\title{
Mitochondrial permeability regulates cardiac endothelial cell necroptosis and cardiac allograft rejection
}

Ingrid Gan, The University of Western Ontario

Supervisor: Zhang, Zhu-XU, The University of Western Ontario

Joint Supervisor: Jevnikar, Anthony, The University of Western Ontario

A thesis submitted in partial fulfillment of the requirements for the Master of Science degree in Pathology

(C) Ingrid Gan 2018

Follow this and additional works at: https://ir.lib.uwo.ca/etd

\section{Recommended Citation}

Gan, Ingrid, "Mitochondrial permeability regulates cardiac endothelial cell necroptosis and cardiac allograft rejection" (2018). Electronic Thesis and Dissertation Repository. 5919.

https://ir.lib.uwo.ca/etd/5919

This Dissertation/Thesis is brought to you for free and open access by Scholarship@Western. It has been accepted for inclusion in Electronic Thesis and Dissertation Repository by an authorized administrator of Scholarship@Western. For more information, please contact wlswadmin@uwo.ca. 


\begin{abstract}
Following transplantation, graft rejection continues to be a significant cause of negative patient outcomes. Programmed cell death events are, in turn, significant contributors to the delayed function and rejection of transplanted organs. We have previously demonstrated that inhibition of necroptosis prevents murine microvascular endothelial cell (MVEC) death and can attenuate murine graft rejection. In this study, we examined the mitochondrial permeability transition pore (mPTP) and its regulator molecule, cyclophilin-D (Cyp-D). Opening of the mPT pore triggers apoptotic molecules release and ultimately results in cell death. However, the role of $\mathrm{MPTP}$ in the necroptotic pathway and in transplantation rejection remains unclear. Here we found that TNF $\alpha$ triggered MVEC to undergo receptor-interacting protein kinase family (RIPK1/3)-dependent necroptosis. Interestingly, the inhibition of either mPTP opening or Cyp-D protected MVECs from necroptosis; inhibition or deficiency of Cyp-D alone attenuated RIPK3-downstream mixed lineage kinase domain like protein (MLKL) phosphorylation. Furthermore, Cyp-D-deficient cardiac grafts showed prolonged survival in allogeneic BALB/c mice post transplantation in comparison to wild- type grafts. Our study suggests that the mPTP may be an important mechanistic mediator of necroptosis in cardiac grafts, and that targeting its opening via the inhibition of Cyp-D presents therapeutic potential in the mitigation of cell death and cardiac graft rejection.
\end{abstract}




\section{Keywords}

Necroptosis, endothelial, heart transplantation, MPTP, Cyp-D 


\section{Acknowledgments}

I would like to thank my supervisors, Dr. Zhu-Xu Zhang and Dr. Anthony Jevnikar for their inspirational guidance and mentorship throughout my study. Many thanks to the lab technicians, Xu-Yan Huang, Ziqin Yin, and Winnie Liu for their assistance; to the microsurgery team, Jifu Jiang and Dameng Lian for murine heterotopic cardiac transplantation; and the administrative staff, Pamela Gardner, Punnya Bose, and Catherine Fraser for their support. Thank you to Dr. Aaron Haig for lending his expertise on pathology, and finally, thank you to my advisory committee members Dr. Martin Dunnewald and Dr. Lakshmann Gunaratnam for providing vital insights to this project. 


\section{Table of Contents}

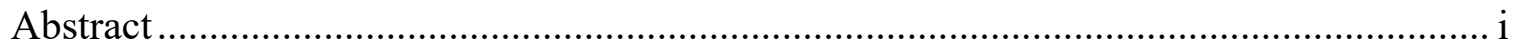

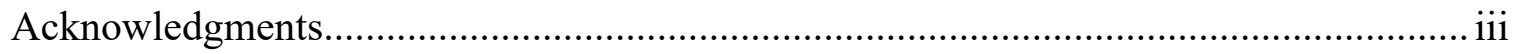

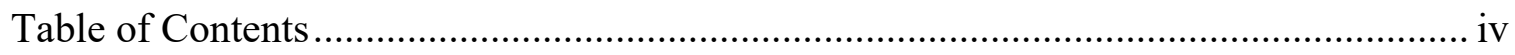

List of Abbreviations .......................................................................................... vi

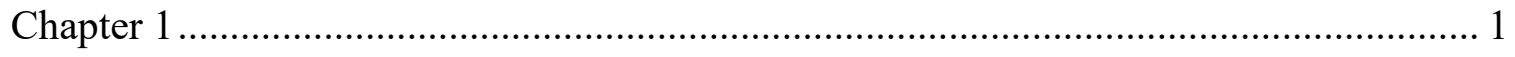

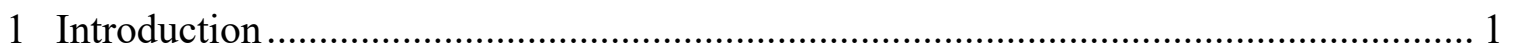

1.1 Current challenges in cardiac transplant rejection ....................................... 1

1.2 Involvement of endothelial cells in heart failure ........................................... 1

1.3 Pathways of endothelial cell death............................................................... 2

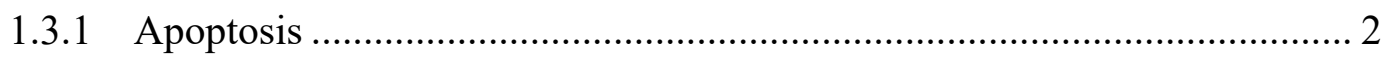

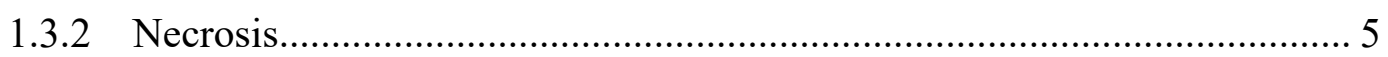

1.4 Mitochondria regulated cell death .............................................................. 7

1.4.1 Mitochondria in endothelial cell death ............................................. 7

1.4.2 Mitochondria plays a role in apoptosis and necroptosis ......................... 7

1.4.3 BCL-2 family regulated mitochondrial dysfunction and apoptosis ............. 9

1.4.4 Mitochondrial permeability transition dysfunction ............................... 9

1.5 The role of cyclophilin in mitochondrial physiology ..................................... 12

1.5.1 The role of cyclophilin in permeability transition ............................... 12

1.5.2 The role of cyclophilin in immune regulation ................................... 12

1.6 Are mitochondria directly involved in programmed necrosis? ........................ 13

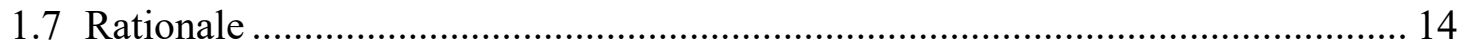

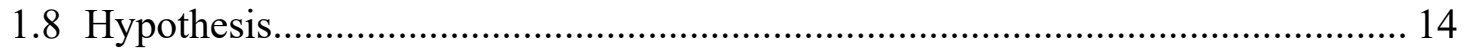

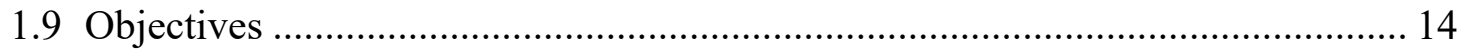

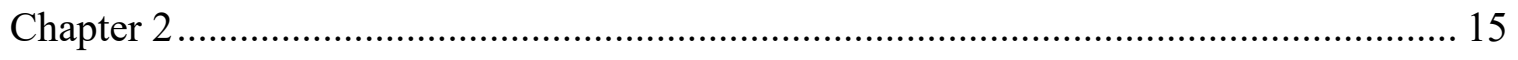

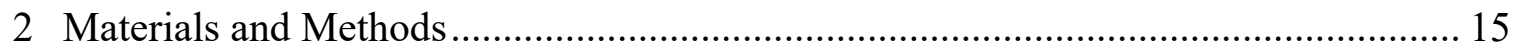




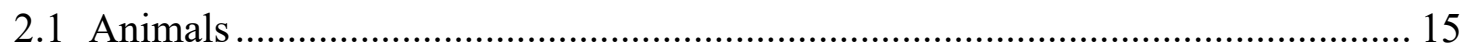

2.2 Heterotopic cardiac transplantation and post-operative monitoring ..................... 15

2.3 Histology and Immunohistochemistry ........................................................... 15

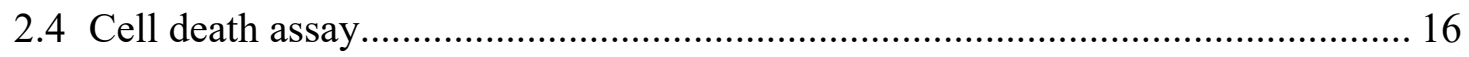

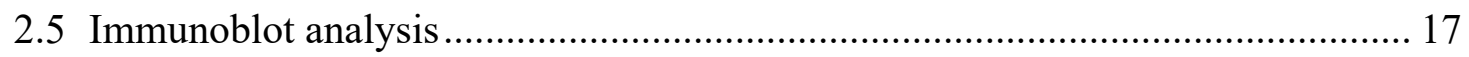

2.6 shRNA-mediated Cyp-D RNA silencing and Real-time PCR............................ 17

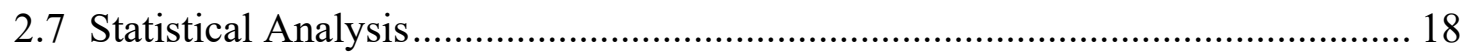

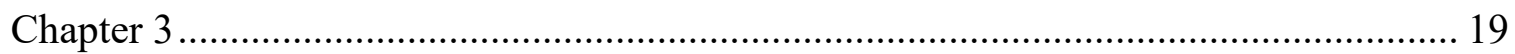

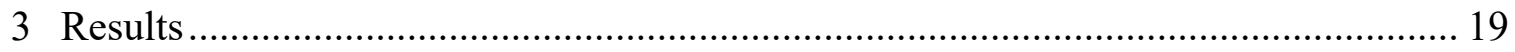

3.1 Mitochondrial permeability participates in MVEC necroptosis ........................... 19

3.2 ROS and Caspases-3 and -9 do not contribute towards MVEC necroptosis ........ 25

3.3 Cyp-D mediated necroptosis is linked to MLKL activation ................................ 28

3.4 Cyp-D deficiency in donor cardiac grafts attenuates rejection ............................. 31

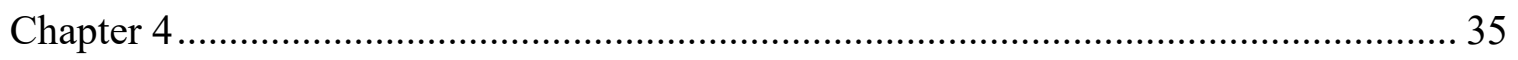

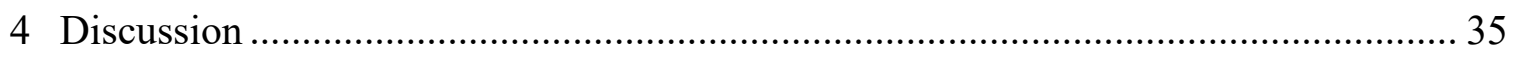

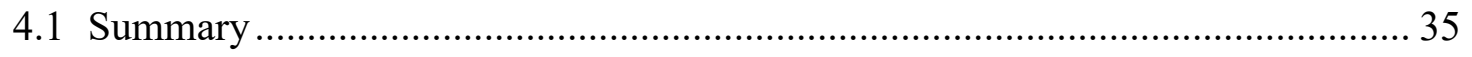

4.2 MVECs necroptosis is mediated by mitochondrial dependent mechanisms ........ 35

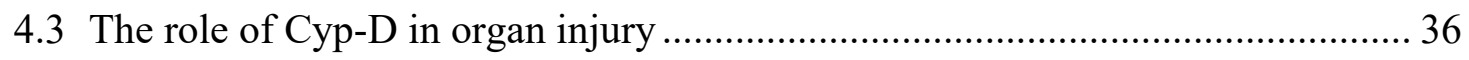

4.4 Mediators of RIPK1/3/MLKL and mitochondrial-dependent MVEC necroptosis37

4.5 Strategy to prevent graft injury in transplantation .............................................. 38

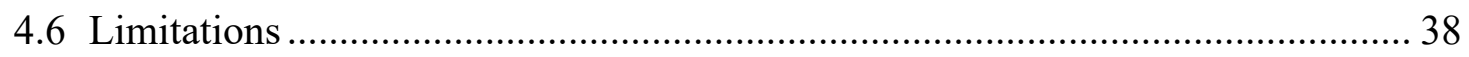

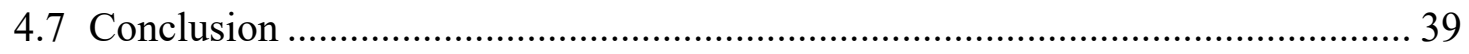

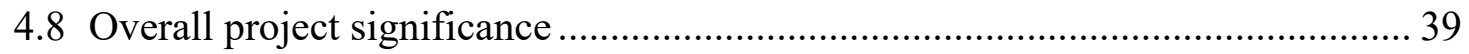

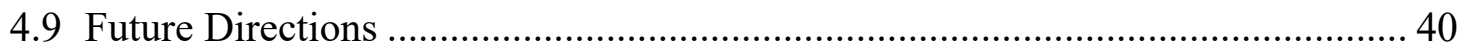

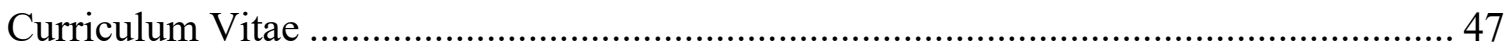




\section{List of Abbreviations}

CsA: Cyclosporin A.

Cyp-D: Cyclophilin-D.

DEVD: Z-Asp-Glu-Val-Asp- Fluoromethylketoe.

HMGB1: high mobility group box 1 .

hTNF $\alpha$ : human tumor necrosis factor alpha.

IETD: Z-Ile-Glu-Thr-Asp-Fluoromethylketoe.

LEHD: Z-Leu-Glu-His-Asp-Fluoromethylketoe.

pMLKL: phosphorylated mixed lineage kinase domain like protein.

MVEC: mouse microvascular endothelial cells.

mPTP: mitochondrial permeability transition pore.

Nec-1s: Necrostatin-1s;

PCR: polymerase chain reaction.

RIPK: receptor interacting protein kinase.

ROS: reactive oxygen species.

shRNA: short hairpin RNA.

TNFR: tumor necrosis factor. 


\section{Chapter 1}

\section{Introduction}

\subsection{Current challenges in cardiac transplant rejection}

For patients with chronic heart failure, heart transplantation is typically the preferred therapy. However, despite an improvement in the one-year survival rate of heart transplant recipients of up to $90 \%$ by 2016 , there has been no significant improvement in the longer-term mortality rate beyond the first year, where factors including malignancy, antibody-mediated rejection, and cardiac allograft vasculopathy result in most cardiac death. ${ }^{1,2}$ Post-transplantation injury is associated with various forms of programmed cell death (PCD) that may severely compromise cardiac tissue viability, engage in innate and adaptive immune responses, and promote inflammation to further exacerbate injury to the graft. As long-term survival of organs has not been greatly improved by solely focusing on recipient immunoregulatory mechanisms, developing new clinical treatment strategies that target PCD and reduce inflammation in transplant organs, in conjunction with the continued use of existing therapies involving immunosuppressants, may be crucial for prolonging graft survival. ${ }^{3,4}$

\subsection{Involvement of endothelial cells in heart failure}

The endothelial cells that line the vascular endothelium perform critical roles in maintaining vascular homeostasis. Some common functions include: regulating smooth muscle tone, exchanging fluid and macromolecules between blood and surrounding tissues, and participating in immune surveillance. ${ }^{5}$ Upon endothelial cell injury due to innate or environmental stress post transplantation, vascular lesions occur. These vascular lesions may progress from progressive thickening of the arterial intima layer to formation of fatty plaque deposits and may, ultimately, result in heart failure.

One of the leading causes of endothelial cell injury is cell death, typically apoptosis, where activation of intracellular proteases, named caspases, induce cells to undergo nuclear fragmentation and formation of apoptotic bodies on the cellular surface. ${ }^{6,7}$ This differs from necrosis, which is characterized by cellular swelling and 
rupture of the plasma membrane accompanied by inflammation. ${ }^{8}$ Here we discuss the specific mechanistic pathways of cell death that occur in endothelial cells.

\subsection{Pathways of endothelial cell death}

Post-transplant graft injury can be triggered by various form of PCD, including necrosis and apoptosis. Whereas necrosis includes "accidental" types of death which result in the release of pro-inflammatory molecules, apoptosis is primarily mediated by a cascade of caspase inducing double-stranded DNA breaks and resulting in plasma membrane blebbing. Two independent apoptotic signaling cascades - the extrinsic and intrinsic pathways - have been identified.

\subsubsection{Apoptosis}

\section{Extrinsic apoptosis}

The extrinsic apoptotic pathway is triggered by the binding of first apoptosis signal receptor (Fas) or tumor necrosis factor receptor 1(TNFR 1). The Fas/Fas ligand complex then recruits death domain-containing protein (FADD), pro-caspase 8 , cellular inhibitor of apoptosis protein (cIAPs), and cFLIPs to form the death-inducing signaling complex (DISC). The DISC complex directly cleaves and activates pro-caspase 8 , which then triggers the activation of pro-caspase 3 , the definitive enzyme for the execution of apoptosis, promoting cytosolic and nuclear alterations for cell disassembly. Caspase 3 activates the caspase-activated DNase (CAD) through cleavage of the inhibitor of CAD (ICAD), which then promotes double stranded DNA breaks and results in DNA fragmentation ${ }^{7,9}$ (Fig 1).

\section{Intrinsic apoptosis}

Intrinsic apoptosis is controlled by mitochondrial enzymes ${ }^{9}$. After a cell is stimulated by extracellular or intracellular stressors, pro-apoptotic members of the Bcl-2 protein family such as BAX and BAK accumulate at the mitochondria, resulting in mitochondrial outer membrane permeabilization (MOMP) and the release of cytochrome $c$ and other pro-apoptotic mitochondrial molecules. Cytochrome $c$ reacts with apoptotic peptidase activating factor (APAF-1) and dATP to form the apoptosome complex ${ }^{10,11}$. 
This multiprotein complex activates caspase 9, which in turn cleaves effector caspases 3, 6, and 7. Mature caspases 3 and 7 cleave a large set of substrates resulting in the morphological hallmarks of apoptosis, such as nuclear condensation, phosphatidylserine exposure, and the release of membrane bound vesicles without cell membrane permeabilization ${ }^{12,13}$. Similarly, opening of the mitochondrial pore stimulates the release of apoptosis-inducing factor (AIF) and endonuclease G (EndoG) into the nucleus, which triggers chromatin condensation and DNA fragmentation. ${ }^{14}$ 


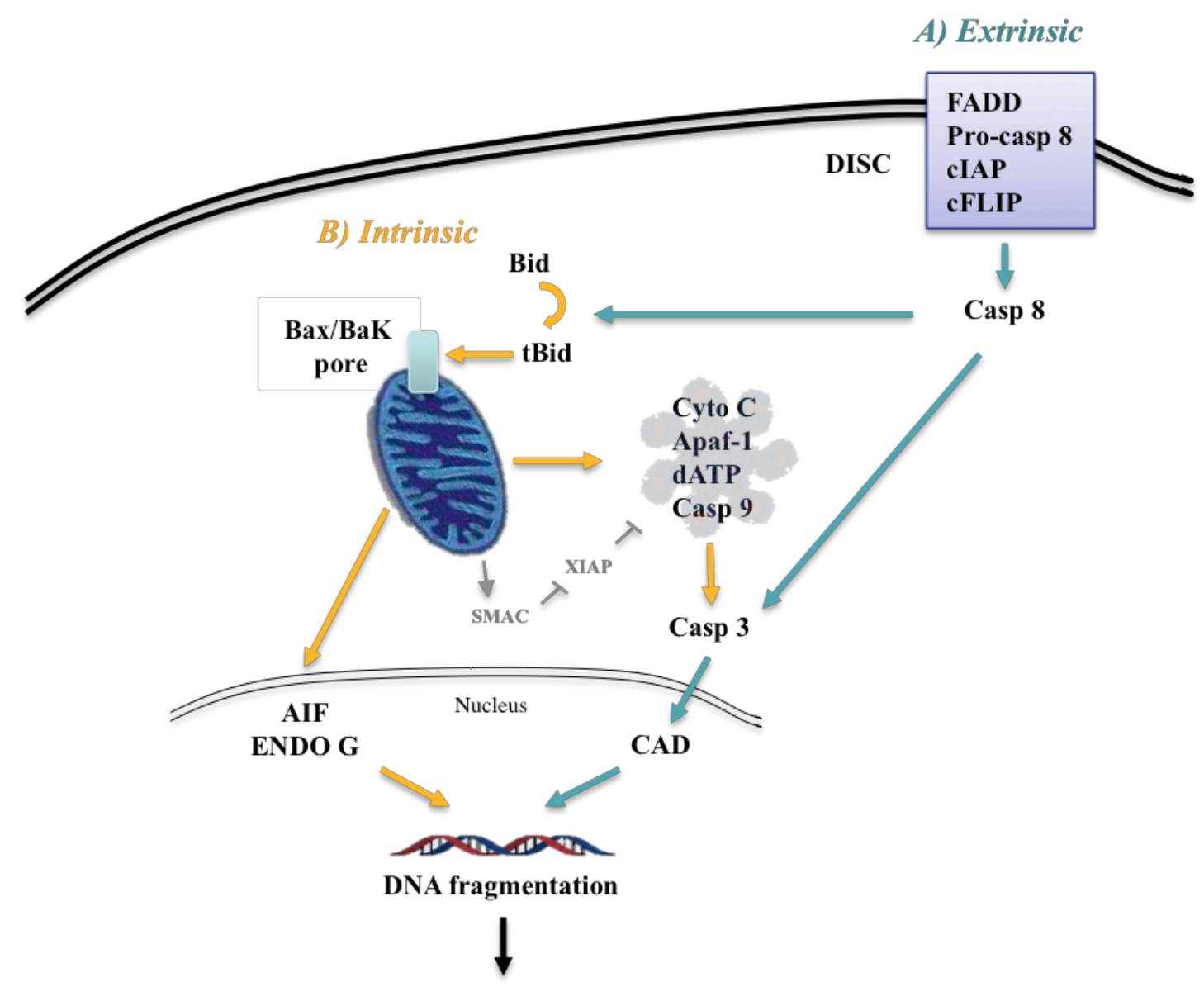

Apoptosis

Fig 1. Extrinsic vs intrinsic apoptotic pathway Apoptosis can be categorized into the extrinsic and intrinsic pathway. The A) extrinsic pathway can be stimulated by TNF receptor superfamily cytokines such as FAS, TRAIl, or TNF $\alpha$ that result in the aggregation of FADD and caspase 8 complex. Caspase 8 initiates the downstream effector caspase 3 cleavage. Activated caspase 3 then triggers ICAD cleavage to release the active $\mathrm{CAD}$ nuclease to promote DNA fragmentation. In contrast, the $\mathrm{B}$ ) intrinsic apoptotic pathway involves the mitochondrial pathway upon stimulation of stress signals, triggering pro-apoptotic Bcl-2 protein family to accumulate in the mitochondrial pore, promoting MOMP and causing the release of cytochrome $c$, apoptosis inducing factor (AIF) and ENDO G, or Smac/DIABLO. Cytochrome $c$ forms the apoptosome complex along with Apaf-1/dATP/caspase 9 to trigger caspase 3 activation, and Smac/DIABLO enhance apoptosis by inhibiting XIAP, where XIAP prevents caspase activation. 


\subsubsection{Necrosis}

Necrosis is traditionally considered to be the primary form of inflammatory cell death. Instead of the characteristic chromatin condensation, cell shrinking, and membrane blebs in apoptosis, necrosis is defined by increased cell volume, organelle swelling, lysosomal membrane permeabilization, and plasma membrane disruption. ${ }^{4,15}$ This results in the leakage of non-membrane enclosed cytoplasmic contents including proinflammatory endogenous molecules such as heat shock proteins (HSP), high mobility group box 1 (HMGB1), and uric acid, any of which can interact with the immune cells of the transplanted organ. ${ }^{16}$ Multiple forms of necrotic-like cell death exist that display these morphological hallmarks.

\section{Necroptosis}

Necroptosis is currently the most investigated pathway of regulated necrosis. This regulated pathway features the necrotic morphology, but is mediated by receptorinteracting protein kinase (RIPK) and can be inhibited by Necrostatin-1 (Nec-1). Necroptosis is induced by death receptors that include Fas and TNF $\alpha$ receptor. TNF $\alpha$ is a pro-inflammatory molecule with important immunoregulatory roles in mammalian immunity and cellular homeostasis. ${ }^{15,16}$

Upon stimulation of TNFR by TNF $\alpha$, TNFR associated death domain (TRADD) is recruited to the plasma membrane, which in turn recruits RIPK1, cellular inhibitors of apoptosis protein-1 and 2 (cIAP1/2), linear ubiquitin chain assembly complex (LUBAC), and TNF receptor-associated factor 2 and 5 (TRAF2/5) to form a receptor-bound complex I that initiates downstream signaling events ${ }^{16,17}$. The polyubiquitination of RIPK1 results in nuclear factor- $\kappa \mathrm{B}(\mathrm{NF}-\kappa \mathrm{B})$ activation, leading to cell survival and the release of pro-inflammatory cytokine. On the other hand, the deubiquitination of RIPK1 promotes cell death by associating with RIPK3, causing complex I to dissociate from the membrane, forming a cytosolic complex II with recruitment of FADD and caspase 8 . The activation of caspase 8 will initiate apoptosis by cleaving RIPK1 and RIPK3, whereas its inactivation by endogenous cFLIP-L or pharmacological caspase inhibitor (Z-IETD-fmk) allows the formation of RIPK1-RIPK3 necrosome complex IIb. Activated necrosome 
complex then recruits mixed-lineage kinase domain-like protein (MLKL) that ultimately induces cell rupture and necroptosis. ${ }^{17,18,19}$

\section{Ferroptosis and oxytosis}

Ferroptosis is an iron dependent form of PCD characterized by decreased mitochondrial membrane densities, reduction of mitochondria crista, and eventual mitochondrial membrane rupture. ${ }^{20,21}$ Mechanically, the small molecule ferroptosis inducer erastin inhibits the amino acid transporter system Xc-Cys/Glu antiporter which allows extracellular L-Cys and intracellular L-Glu exchange. This process leads to lipid peroxidation accumulation and increase in iron metabolite ROS. Glutathione (GSH) peroxidase 4 (GPX4), HSP, and nuclear factor erythroid 2 related factor 2 negatively regulate ferroptosis by limiting ROS production and cellular Fe uptake, whereas positive regulators such as NAPH oxidase and tumor protein p53 promote ROS mediated lipid peroxidation. $^{8,22}$

Similarly, inhibition of Xc-Cys/Glu antiporter results in oxytosis, where downstream activation of 12-lipoxygenase initiates mitochondrial ROS and cyclic GMP (cGMP). cGMP induces cGMP channel on the plasma membrane, stimulating $\mathrm{Ca}^{2+}$ influx and lysosomal membrane permeabilization. Through inhibition of Xc-Cys-glu antiporter, ferroptosis and oxytosis utilize iron and calcium metabolism - respectively - to induce regulated necrosis. ${ }^{8,23}$

\section{Parthanatos}

Parthanatos, named after "Thanatos", the personification of death in Greek mythology, involves the accumulation of poly (ADP-ribose) polymerase (PARP) and nuclear translocation of mitochondrial-associated apoptosis-inducing factor (AIF), leading to large-scale fragmentation of DNA and cell death. ${ }^{24,25}$ The process depends on the PARP activation which depletes cellular energy through reduction of nicotinamide adenine dinucleotide $\left(\mathrm{NAD}^{+}\right)$, an essential co-factor of glycolysis and TCA cycle. Genetic deletion of poly-ADP-ribose polymerase-1 (PARP) demonstrates PARP plays an important role in genotoxic stress, diabetes and neurodegeneration experimental models, considering the fact that energy depletion is an important contributor in neuron and 
astrocyte cell death. Under normal physiological conditions, PARP1 localizes in the nucleus and responds to DNA damage. If DNA damage is severe, however, overactivation of PARP promotes parthanatic cell death. ${ }^{26}$ Studies have shown PAR induces AIF release from the mitochondria and translation to the nucleus upon their physical interaction ${ }^{26,27}$, leading to cell death, and the implication that, along with manifesting through the necrotic pathway, apoptotic mechanisms also contribute to parthanatic cell death.

\subsection{Mitochondria regulated cell death}

\subsubsection{Mitochondria in endothelial cell death}

As frontline defenders against vascular disease, microvascular endothelial cells not only power the cardiomyocytes with oxygen delivery and energetic substrates, but also play a role in preventing cardiovascular diseases post transplantation such as atherosclerosis and cardiac vasculopathy. ${ }^{28}$ Furthermore, endothelial cells play a crucial role in modulating mitochondrial function in the heart where endothelial cells outnumber cardiomyocytes by nearly 3-fold. ${ }^{5}$ As proteins involved in mitochondrial homeostasis can be involved in both apoptosis and necroptosis, it is important to place an emphasis on better understanding the mechanisms of mitochondrial regulated cell death in order to reduce injury an improve function in cardiac tissue.

\subsubsection{Mitochondria plays a role in apoptosis and necroptosis}

Mitochondria are known foremost as the primary cellular- energy- producing organelles, but they also play a central role in cell death. Activation of death receptors can lead to mitochondrial dysfunction events that can induce both apoptosis and necroptosis. ${ }^{29,30}$ Stimulation of either death pathway depends on the availability of caspases - recruitment of adaptor proteins, such as TRADD, will lead to activation of caspase 8 and cleavage of B-cell lymphoma protein BID to induce mitochondrial pore permeabilization. This permeabilization results in DNA fragmentation and apoptosis; in the absence or inhibition of caspase 8 , however, the TRADD adaptor protein complex recruits RIPK1, which forms the necrosome complex with RIPK3. The RIPK1/3 complex, in turn, recruits MLKL, resulting in plasma membrane rupture and necroptosis. 


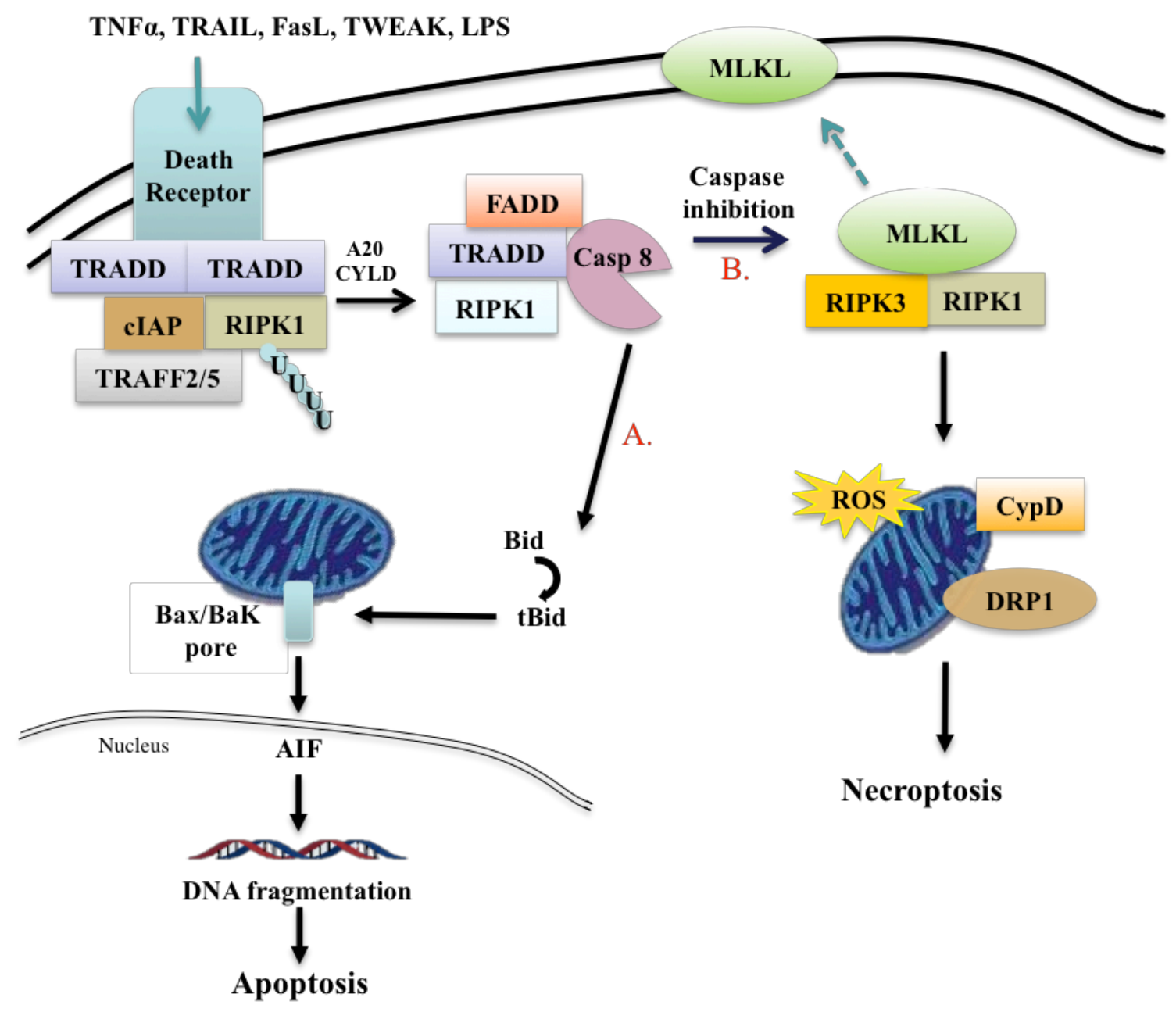

Fig 2. Mitochondria participates in apoptosis and necroptosis

Upon stimulation by TNF $\alpha$ or other ligands (TRAIL, FasL, TWEAK, LPS), an inner membrane adaptor protein complex is formed along with RIPK1. Deubiqutinated RIPK1 leads to A) caspase 8 activation and Bid cleavage by caspase 2, which leads to the opening of $\mathrm{Bax} / \mathrm{BaK}$ pore in the mitochondrial membrane, subsequent AIF activation, DNA fragmentation, and apoptosis. Under conditions where B) caspase 8 is inhibited, RIPK1 and RIPK3 form the necrosome complex with MLKL. MLKL can be targeted to both the plasma membrane to induce membrane rupture, or to the mitochondria triggering ROS and necroptosis. 


\subsubsection{BCL-2 family regulated mitochondrial dysfunction and apoptosis}

The B-cell lymphoma 2 (BCL-2) family proteins mediate mitochondrial apoptosis and cell survival. The pro-apoptotic members include BCL-2 associated X protein (BAX), BCL-2 homologous antagonist killer (BAK), BCL-2 interaction death promoter (BAD), BH3 interacting-domain death agonist (BID), and BCL-2 interaction mediator of cell death (BIM). ${ }^{31}$ Following a death signal, the pro-apoptotic members translocate to the outer mitochondrial membrane (OMM) to be activated by BID, inducing permeabilization of the OMM. This permeabilization stimulates the release of proapoptotic factors such as cytochrome $c$ from their inner membrane space. The cytosolic cytochrome $c$ induces the formation of the apoptosome that activates effector caspases to initiate apoptosis ${ }^{31,32}$

\subsubsection{Mitochondrial permeability transition dysfunction}

The mitochondrial permeability transition pore (mPTP) is a protein complex suggested to span both the outer and the inner mitochondrial membranes, and is presumed to perform important physiological roles. ${ }^{33,34}$ The precise molecular composition of this pore remains highly controversial, but is thought to comprise of the voltage-dependent anion channel (VDAC) on the outer membrane and adenine nucleotide translocase (ANT) on the inner membrane (30), spastic paraplegia 7 (SPG7), phosphate carrier (PiC), ATP synthase, and cyclophilin D (CypD). ${ }^{35}$ Recently, the role of VDAC and ANT was questioned, where mitochondrial PT deficient in VDAC and ANT still led to MPT onset. ${ }^{36}$ Furthermore, sensitivity to MPT inhibitor CsA (cyclosporine A) was still displayed in VDAC and ANT null mitochondria. ${ }^{36}$

In normal conditions, the inner membrane channel opening is regulated to prevent membrane potential dissipation and proton gradient loss. When stimulated by intracellular signals unrelated to cell death, brief opening of mPTP (described as “flickering") causes transient mitochondrial membrane potential variations necessary for cellular homeostasis such as mitochondrial $\mathrm{Ca}^{2+}$ extrusion during normal conditions. ${ }^{37}$ During $\mathrm{Ca}^{2+}$ overload and oxidative stress conditions, however, prolonged opening of the mPTP is maintained, which promotes mitochondrial swelling, membrane potential loss, ATP depletion, and, eventually, mitochondrial membrane permeabilization (OMM), 
which may result in cell death (30-34). Activation of OMM allows the release of the proapoptotic factors such as cytochrome $c$ into the cytosol (31). These events are regulated by the Bcl-2 protein family; pro-apoptotic proteins Bax and Bak allow membrane permeabilization, while the anti-apoptotic members inhibit this process. ${ }^{32,38,39}$

The involvement of mitochondria, MPTP, and Bax/Bak as downstream necroptotic mediators is still a point of contention. It has been suggested that mitochondria play a role in regulated necrosis, where Bax/Bak generate a level of permeability in the outer mitochondrial membrane in non-oligomerized forms ${ }^{40}$ In addition, it has been shown that necroptosis induced by DNA damaging agent (MNNG) is dependent on Bax activation. It is possible that besides promoting apoptosis, the Bcl-2 family proteins may be required for necroptotic induction..$^{41,42}$ 


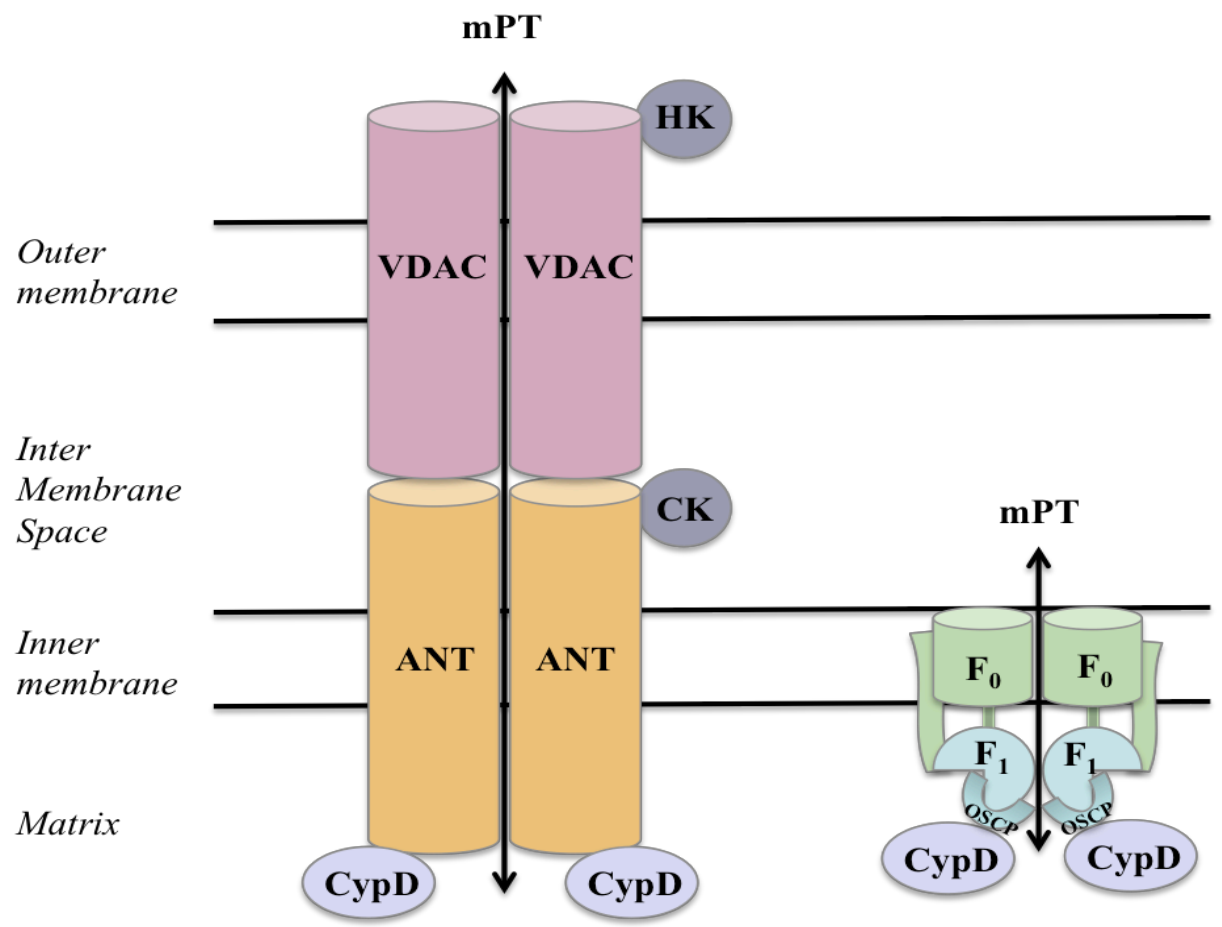

\section{Fig 3. Molecular components of MPTP}

mPTP plays an important role in cell death by opening up the mitochondrial membrane through the formation of transporter channel proteins. Under apoptotic stimuli, prolonged pore opening allows for entry of small molecules, such as $\mathrm{Ca}^{2+}$ and protons, causing a loss of potential, osmotic imbalance, and mitochondrial swelling.

mPTP is thought to be composed of voltage dependent anion channel (VDAC), adenine nucleotide translocator (ANT), peripheral benzodiazepine receptor (PBR), hexokinase (HK), creatine kinase (DK), and cyclophilin D (Cyp-D). More recent research has suggested that the VDAC and ANT may not be necessary for the mPT process, It is suggested that MPTP complex now constitutes dimers of F0-F1 adenosine triphosphate (ATP) synthase regulated by CypD, where CypD associates with the $\mathrm{F} 1$ component oligomycin sensitivity-conferring protein (OSCP)to trigger pore opening, and that this process is inhibited by CsA. ${ }^{43}$ 


\subsection{The role of cyclophilin in mitochondrial physiology}

\subsubsection{The role of cyclophilin in permeability transition}

Although an agreement on the precise MPT pore components is yet to be reached, the soluble protein $\mathrm{CypD}$ is the only unequivocally-established component of the pore complex to date. ${ }^{44} \mathrm{CypD}$ is a peptidylprolyl isomerase that is critical in regulating MPTP opening. CypD resides in the mitochondrial matrix, which translocates to the inner mitochondrial membrane during oxidative stress. CypD-deficient mitochondria were resistant to calcium overload and protective against cardiac ischemia reperfusion injury, whereas increased CypD level results in excessive ROS generation following ischemia injury in rats. ${ }^{45}$

CypD is then suggested to mediate a conformational change in the adenine nucleotide translocase and triggers mPTP opening, mitochondrial swelling, and release of apoptotic effectors. ${ }^{46}$ Recently, it is proposed that CypD binds to ATP synthase, the rotary enzyme responsible for ATP synthesis. The lateral stalk links the catalytic $\mathrm{F}_{1}$ and the membrane bound $\mathrm{F}_{0}$ portion together. More specifically, it is proposed that CypD may directly interact with the OSCP subunit of the lateral stalk, where, following separation of individual lateral stalk subunits, $\mathrm{CypD}$ was found exclusively in the immunoprecipitation with OSCP antibody. ${ }^{47,48}$

\subsubsection{The role of cyclophilin in immune regulation}

Aside from directly regulating the MPTP pore, Cyclophilin is also important in regulating immune function through its interaction with cyclosporine $(\mathrm{CsA}) .{ }^{49} \mathrm{CsA}$ is a cyclic polypeptide metabolized by a number of fungi, and its effective immunosuppressive properties revolutionized the field of transplantation since its discovery in 1972. ${ }^{49,50}$ Binding of CsA with Cyclophilin A inhibits calcineurin function a calcium/calmodulin-dependent serine threonine protein phosphatase that activates the $\mathrm{T}$ cell promoter IL-2 and inhibits the overall immune response. ${ }^{52}$ More specifically, calcineurin inhibition prevents its substrate phospho-NFAT dephosphorylation and translocation to the nucleus - a process necessary for IL-2 activation and subsequent T cell activation. ${ }^{51}$ 


\subsection{Are mitochondria directly involved in programmed necrosis?}

The involvement of mitochondria and MPTP as downstream mediators in the necroptosis pathway is still a point of contention. While it is well-established that mitochondria are the platforms for apoptosis execution, the prospect of their direct involvement in programmed necrosis remains controversial, and contradictory experiments point towards either mitochondrial-dependent or-independent forms of necrosis or necroptosis.

\section{Evidence for the role of mitochondria in necroptosis:}

Many studies have suggested that necrosome signalling might involve ROS generation from the mitochondria. ${ }^{53}$ In addition, studies demonstrated necrotic death was inhibited in endothelial cells with the mitochondrial antioxidant MnSOD. ${ }^{54,55}$ RIPK1, RIPK3, and/or MLKL are translocated to the mitochondria during necroptosis and thus result in ROS production. ${ }^{56}$ In particular, RIPK3 recruits MLKL to the necrosome complex which is suggested to promote MLKL translocation to the mitochondria to induce cell death - where MLKL expression is increased in mitochondrial fraction following treatment of TNF $\alpha$ and pan-caspase inhibitor.

Genetics experiments confirmed the involvement of mPTP opening during PCD where CyP-D KO in MEFs (mice embryonic fibroblast cells) resulted in a partial rescue of necroptotic cell death 57,58 in vitro, ${ }^{57,58}$ and that $\mathrm{mPT}$ pore is primarily involved in necrotic cell death instead of apoptosis. ${ }^{59}$ In addition, studies have shown that siRNA mediated knockdown of mitochondrial fission molecule Drp1 were able to inhibit TNF $\alpha$ mediated necroptosis in HeLa and HT-29 cells. ${ }^{60}$ Furthermore, Drp1 depletion decreased death in rat renal tubule epithelial cells after TNF $\alpha$ treatment. ${ }^{61}$ These studies all suggest that mitochondria play a pivotal role in the execution of the necroptotic pathway.

\section{Evidence against the role of mitochondria in necroptosis:}

While studies demonstrated the role of mitochondrial dysfunction in necroptosis, others suggested that mitochondria might be dispensable for this process. A previous study revealed that, after mitochondrial depletion by mitophagy in SVEC and 3T3 cells, 
necroptosis was still observed. ${ }^{62}$ Another study also questioned the downstream RIPK3mitochondrial association, where RIPK 3 or CypD ablation were protective individually, but double knockout mice exhibited even greater protection, ${ }^{46}$ suggesting that the necrosome and the MPT pore may be distinct pathways. However, CypD deletion did not further attenuate myocardium ischemia reperfusion injury (IRI) to that conferred by RIPK1 inhibition in the heart, ${ }^{63}$ which raises the question of whether tissue-and cell specific differences may contribute to the coupling of MPT pore to necroptosis.

\subsection{Rationale}

Our previous studies have demonstrated that RIPK3-medidated necroptosis in donor heart and kidney grafts can promote inflammatory injury and transplant rejection, and that the inhibition of RIPK3 expression attenuated necrosis and reduced early graft injury and rejection ${ }^{64,70}$. The down-stream pathway of necroptosis is not clear. It is controversial whether mitochondria are involved in necroptosis. In our current study, we aim to determine the role of CypD-regulated $\mathrm{mPT}$ in necroptosis in cardiac endothelial cells and in mouse heterotopic transplantation model. This will provide rationale to design an effective inhibition strategy to promote transplant tolerance.

\subsection{Hypothesis}

We hypothesize that Cyp-D inhibition can inhibit mPT, thus preventing cell necroptotic death, and can trigger the release of proinflammatory CDAMP, preventing graft injury in heart transplants and thus prolonging graft survival,.

\subsection{Objectives}

1. To determine if the inhibition of CypD function in cardiac endothelial cells can prevent necroptotic death.

2. To determine if CypD deficiency in donor cardiac grafts can inhibit tissue injury and prolong graft survival. 


\section{Chapter 2}

\section{Materials and Methods}

\subsection{Animals}

Male inbred C57BL/6 (B6), BALB/c mice and B6. Cyp-D ${ }^{-/-}$mice (Jackson Lab) were maintained at the animal facility at Western University. All experimental procedures were approved by the Animal Use Committee of Western University.

\subsection{Heterotopic cardiac transplantation and post-operative monitoring}

Donor cardiac grafts were perfused with saline to remove blood after being anesthetized with the mixture ketamine/xylazine according to the approved animal protocol. The inferior vena cava and dorsal aorta were then clamped above and below the cardiac graft. The graft was then removed, and intra-abdominal heterotopic cardiac transplants were performed in our microsurgery laboratory in accordance with an approved protocol. The recipient received sirolimus (rapamycin, LCL laboratories, USA) from day 0 to day 9 post-transplantation ( $1 \mathrm{mg} / \mathrm{kg}$ mouse). Pulsation of the cardiac graft was monitored daily. Cessation of pulsation was defined as the endpoint of rejection.

\subsection{Histology and Immunohistochemistry}

Grafts were collected on day 28, perfused with PBS, cut transversely, and then either frozen using Tissue-Tek ${ }^{\circledR}$ O.C.T. Compound (Sakura ${ }^{\circledR}$ Finetek) or fixed with 5\% formalin for paraffin embedding. Paraffin sections were used for hematoxylin and eosin (H\&E) staining.

All injury scores were evaluated by a pathologist in a blinded manner. Graft injury was evaluated based on change in endothelium as compared with naïve. Damage was scored on a scale of 0-4 (0: no change, 1: 0-24\% change, 2: 25-49\% change, 3: 50$74 \%$ change, $4:>75 \%$ change). 


\subsection{Cell death assay}

B6 and Cyp- $\mathrm{D}^{-/-}$microvascular endothelial cells (MVEC) were isolated and developed as described previously (18-20). Cells were grown in complete Endothelial Growth Media-2 (EGM-2 medium) supplemented with fetal bovine serum and growth factors (Lonza, USA).

Human TNF $\alpha$ (hTNF $\alpha, 100 \mathrm{ng} / \mathrm{ml}$; PeproTech, USA) was used to induce cell death. Smac-mimetic (100 nM; Selleckchem, USA) was added to suppress the function of Inhibitor of Apoptosis Proteins (IAP). This leads to caspase activation and inhibition of RIPK1 polyubiquitination, and promotes apoptotic cell death. Caspase-mediated apoptosis was inhibited by caspase-8 inhibitor Z-Ile-Glu-Thr-Asp-Fluoromethylketoe (IETD, 10-30 $\mu \mathrm{M}$; APExBIO, USA). Caspase-3 inhibitor Z-Asp-Glu-Val-AspFluoromethylketoe (DEVD, 10-30 $\mu \mathrm{M}$;) and capase-9 inhibitor Z-Leu-Glu-His-AspFluoromethylketoe (LEHD, 10-30 $\mu \mathrm{M}, \mathrm{APExBIO}$ ) were used. RIPK1 inhibitor necrostatin-1s (Nec-1s, $10 \mu \mathrm{M}$; Merck Millipore, USA) or MLKL inhibitor GW806742X (50-1000 nM, Synkinase, USA) were added to inhibit necroptosis. Mitochondrial permeability transition events were inhibited with S-15176 (Sigma-Aldrich, USA). Dose of S-15176 $(1-20 \mu \mathrm{g} / \mathrm{ml})$ was optimized and $16 \mu \mathrm{g} / \mathrm{ml}$ was chosen for the study. Cyclosporin A (CsA) and control FK-506 (Sigma-Aldrich) were used for Cyp-D inhibition. Dose of CsA (1-15 $\mu \mathrm{g} / \mathrm{ml})$ was optimized and $10 \mu \mathrm{g} / \mathrm{ml}$ was used for the study. ROS inhibitors are 1-oxyl-2,2,6,6-tetramethyl-4-hydroxypiperidine (Tempol, 1-10 $\mu \mathrm{M}$, Sigma-Aldrich, USA) and N-acetyl-L-cysteine (NAC, 1-10 $\mu \mathrm{M}$, Sigma-Aldrich). Dose was optimized and $10 \mu \mathrm{M}$ was chosen for the study.

MVEC were seeded on 96-well flat-bottomed plates for 24 hours in complete EGM-2 media (Lonza) to generate a confluent mono-layer. Cell death was detected either a measure of SYTOX ${ }^{\circledR}$ Green Nucleic Acid Stain (100 nM, ThemoFisher, USA) and Cell death (fluorescence intensity of SYTOX) was monitored using the IncuCyte ZOOM ${ }^{\circledR}$ System (Essen Bioscience, USA). Cell death also conformed by 7-AAD (eBioscience, USA) and analyzed bythe CytoFLEX flow cytometer (Beckman Coulter, USA). 


\subsection{Immunoblot analysis}

MVEC were grown to a confluent monolayer and treated as described in cell death assay. Cells were trypsinized, centrifuged at $300 \mathrm{~g}$ for 5 minutes and $50 \mu \mathrm{L}$ nuclear lysis buffer (20 mM HEPES, $0.4 \mathrm{mM} \mathrm{NaCl}, 1 \mathrm{mM}$ EDTA, 1 mM EGTA, 1 mM DTT, 1 mM PMSF) was added to each sample followed by a 30 minutes incubation at $37.5^{\circ} \mathrm{C}$. The nuclear fraction was collected by centrifugation at $10,000 \mathrm{~g}$ for 15 minutes at $4{ }^{\circ} \mathrm{C}$.

Equal amount lysates were loaded for gel electrophoresis. Protein was transferred to a nitrocellulose membrane using electrophoresis blotting system (BioRad, USA). 5\% skim milk and $0.1 \%$ Tween-20 in Tris buffered saline was used for blocking.

The phosphorylated MLKL (pMLKL), Phospho S345 MLKL protein, was detected using rabbit anti-mouse pMLKL antibody (Abcam, USA). Protein was visualized using secondary anti-rabbit IgG horseradish peroxidase (HRP)-linked antibody (Cell Signaling Tech. USA) and chemiluminescent substrate (EMD Millipore, USA). Anti- $\beta$-actin antibody (Sigma-Aldrich, USA) was used as loading control.

\section{6 shRNA-mediated Cyp-D RNA silencing and Real-time PCR}

Cyp-D shRNA (Dharmacon, USA) was transfected into MVEC using Lipofectamine (Invitrogen, USA). Puromycin (Sigma-Aldrich, USA) was used for antibiotic selection to eliminate untransfected MVEC. Cyp-D gene silencing was confirmed by real-time PCR.

Total RNA from wildtype or shRNA transfected MVEC was extracted from tissue or cells by Trizol extraction (Invitrogen, USA). cDNA was generated from RNA using Superscript II (ThermoFisher, USA). Primers are: Cyp-D: CTC CAA CTC CAA GAA CCC GC and TAA AAC AAT TCG GCC AAC TCG C; $\beta$-Actin: CCA GCC TTC CTT CCT GGG TA and CTA GAA GCA TTT GCG GTG CA. Real-time quantitative PCR was performed using a PCR reagent mixture (ABM, Canada) and the CFX Connect (BioRad, USA). B-Actin amplification was used as the endogenous control. 


\subsection{Statistical Analysis}

Data was analyzed using the Student's t-test for unpaired values. The Mantel-Cox $\log$ rank test was used to determine graft survival differences. Differences were considered significant when $\mathrm{p}$-value $\leq 0.05$. 


\section{Chapter 3}

\section{Results}

\subsection{Mitochondrial permeability participates in MVEC necroptosis}

We used wild type and RIPK3 deficient endothelial cells to determine the effects of inflammatory cytokines on cell death. TNF $\alpha$, Smac-mimetic, and caspase- 8 inhibitor IETD or RIPK1 inhibitor Nec-ls were added to wild type B6 and RIPK3 ${ }^{-/-}$MVEC cultures. In wild type B6 MVEC, treatment of TNF $\alpha$ increased cell death compared to untreated cells. The addition of caspase- 8 inhibitor IETD enhanced TNF $\alpha$ induced cell death (Figure 4A), suggesting necroptotic death. ${ }^{64}$ Necroptosis in MVEC was inhibited by the addition of RIPK1 inhibitor Nec-1s or RIPK3 deficiency in MVEC (Figure 4A).

To determine if mitochondrial dysfunction events play a role in necroptosis, mitochondria transition permeability inhibitor S-15176 was added to MVEC. S-15176 prevents collapse of the electrochemical gradient across the mitochondrial membrane and inhibits release of apoptotic molecules. ${ }^{65,66}$ We found that the addition of S-15176 to TNF $\alpha+$ IETD treated MVEC significantly reduced necroptosis (Figure 4B).

Opening of mPTP is largely regulated by Cyp-D. Cyp-D may regulate apoptotic cell death as well necrotic cell death. ${ }^{67}$ It has been shown that Cyp-D deficiency in mouse embryonic fibroblast cells resulted in a partial rescue of necroptotic cell death in vitro. ${ }^{57}$ However, another study showed that RIKP1/3 and mPTP/Cyp-D mediate two distinct death pathway. ${ }^{46}$ We studied the role of Cyp-D in MVEC necroptosis. Initially, we used CsA, a calcineurin inhibitor and classical immunosuppressive drug in clinical transplantation that can bind to Cyp-D with high affinity and inhibit mPTP opening. CsA inhibited TNF $\alpha$-induced necroptotic cell death upon simultaneous caspase- 8 inhibition (Figure 1C). In contrast, FK506, a non-Cyp-D binding and calcineurin-inhibiting immunosuppressive drug, did not inhibit cell death (Figure 1C), implying that Cyp-D, but not calcineurin, participates in necroptosis. 
To further confirm the above findings, we developed MVEC from Cyp-Ddeficient mice. Cyp-D in wild type MVEC was silenced using Cyp-D shRNA (Figure 5A). Both Cyp-D silenced MVEC and Cyp-D deficient MVEC resisted TNF $\alpha$-induced necroptosis (Figures 5B\&C). These data support that Cyp-D is a crucial molecule for MVEC necroptosis. 
Figure 4

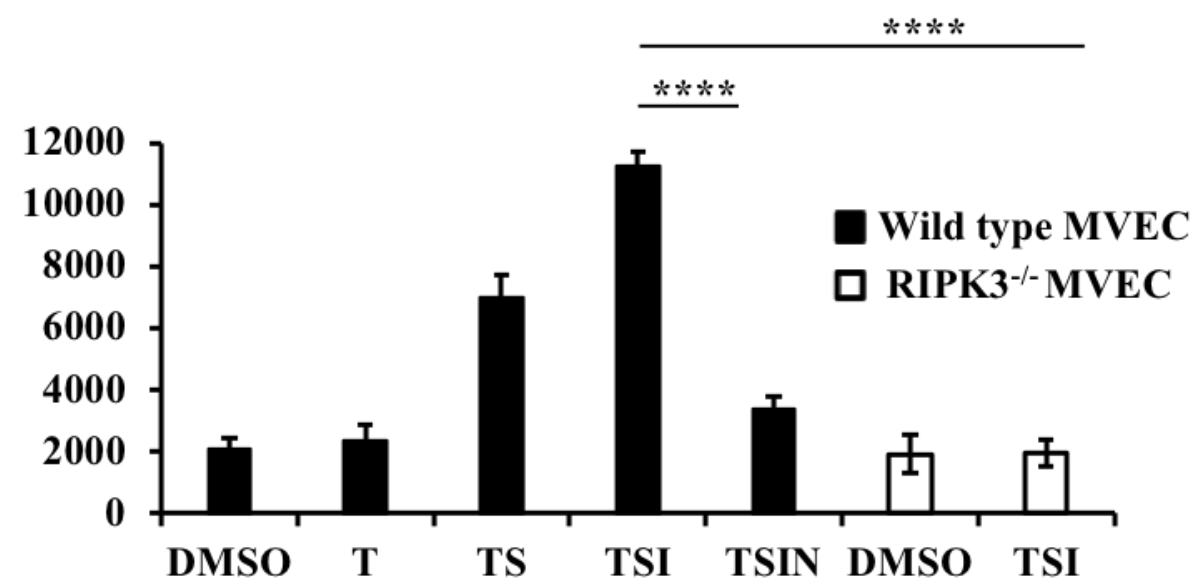

B.

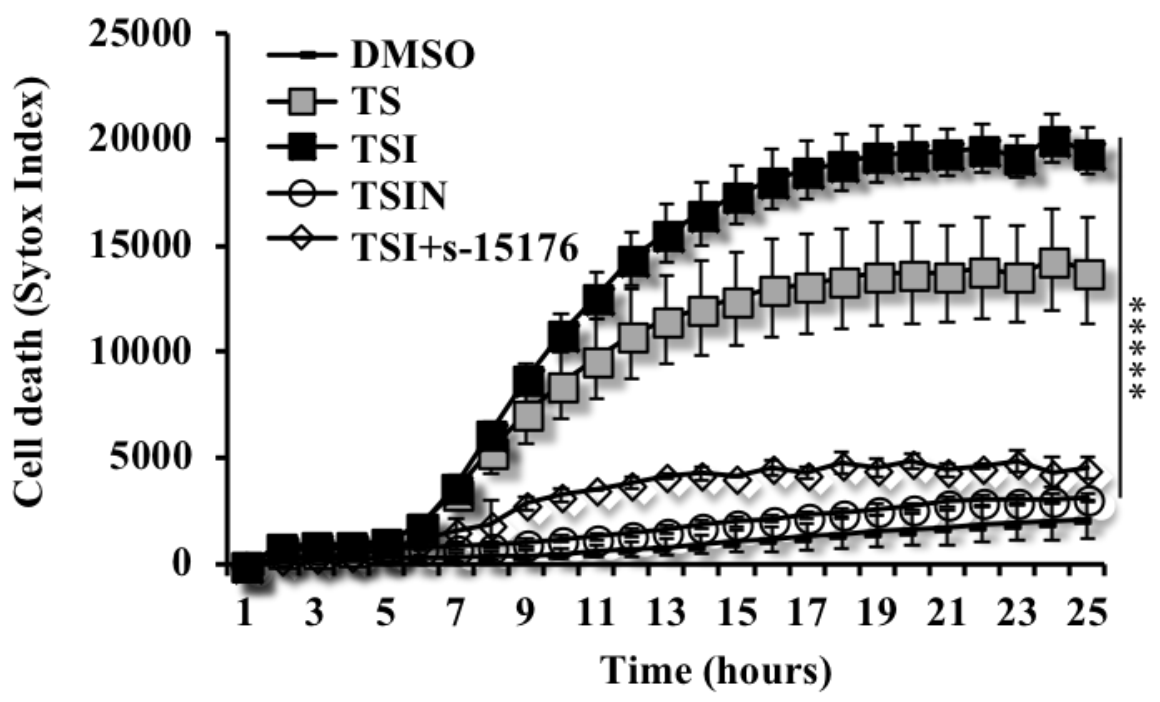

C.

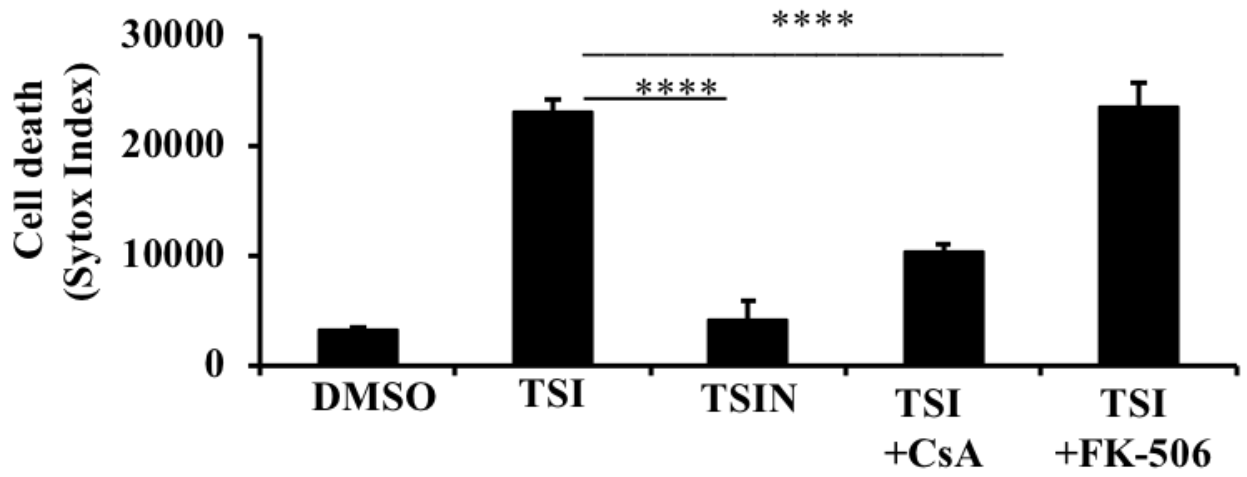


Figure 4. Mitochondrial permeability participates in MVEC necroptosis

(A) B6 MVEC and RIPK3 ${ }^{-/}$MVEC were plated on 96 well plate in triplicates and treated with $100 \mathrm{ng} / \mathrm{mL}$ TNF $\alpha(\mathrm{T}), 10 \mathrm{nM}$ Smac mimetic (S) with or without $30 \mu \mathrm{M}$ IETD(I) and $10 \mu \mathrm{M}$ Nec-1s (N) with addition of SYTOX green. Cell death was quantified by SYTOX fluorescent intensity with IncuCyte ZOOM live imaging system. Data at 24 hours were shown as mean $\pm \mathrm{SD}$ and representative of at least 3 independent experiments. (B) B6 MVEC necroptosis was induced as described above (TSI). mPTP inhibitor S-15176 (16 ug/mL) was added. Cell death (SYTOX green uptake) was monitored for 24 hours by IncuCyte ZOOM live imaging system. Data were shown as mean \pm standard deviation (SD) and representative of 3 independent experiments. (C) CsA (10 ug/ml) and FK506 (10 ug/ml) were added in MVEC prior to necroptosis induction. Data were shown as mean $\pm \mathrm{SD}$ and representative of 3 independent experiments. $* * * * \mathrm{p} \leq 0.0001, * * * * * \mathrm{p} \leq 0.00001, \mathrm{t}$-test. 
Figure 5
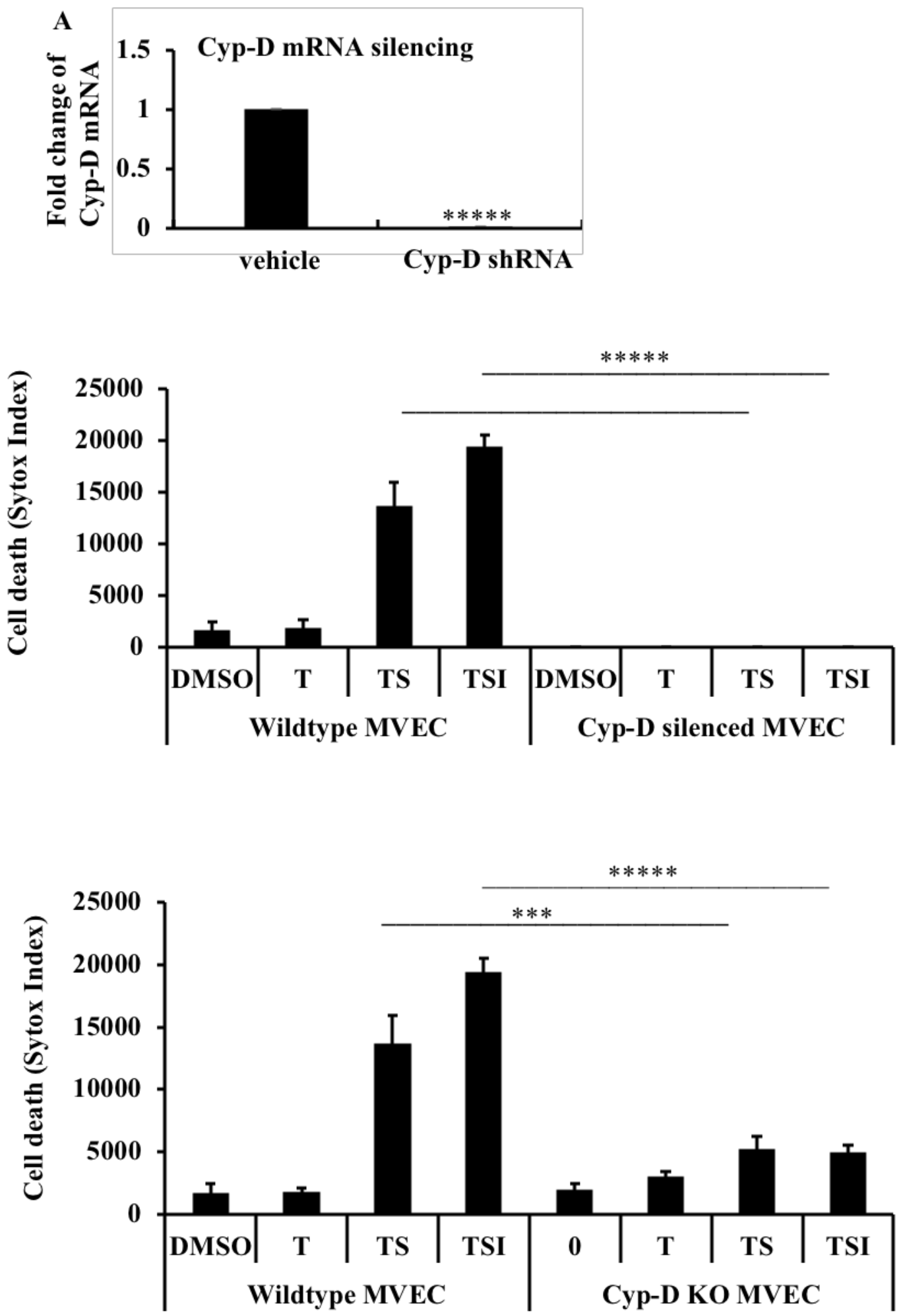


\section{Figure 5. Cyp-D deficiency protects MVEC from necroptosis}

(A) Cyp-D shRNA was transfected into MVEC followed by Puromycin treatment for 2 weeks. Expression of Cyp-D RNA was analyzed by real-time PCR using a PCR reagent mixture (ABM, Canada) and the CFX Connect (BioRad, USA). B-Actin amplification was used as the endogenous control. Data were shown as mean $\pm \mathrm{SD}$ and representative of 3 independent PCR. (B) Cyp-D silenced MVEC were used to induce necroptosis and cell death was quantified as described above. Data at 24 hours were shown as mean $\pm \mathrm{SD}$ and representative of 3 independent experiments. (C) MVEC were isolated from a Cyp-D deficient mouse and then used for the necroptosis assay. Cell death was quantified by SYTOX fluorescent intensity with IncuCyte ZOOM live imaging system. Data at 24 hours were shown as mean \pm SD and representative of 3 independent experiments. $* * * \mathrm{p} \leq 0.001, * * * * * \mathrm{p} \leq 0.00001$, t-test. 


\subsection{ROS and Caspases-3 and -9 do not contribute towards MVEC necroptosis}

Next we studied the down-stream mechanism of Cyp-D mediated necroptosis. Opening of mPTP results in ROS release and activation of caspase- 9 and its downstream caspase-3. Recent studies showed that ROS play a crucial role in necrosis and that inhibition of ROS prevents TNF $\alpha$-induced necroptotic cell death. ${ }^{68,69}$ In our study, addition of caspase-3 inhibitor (DEVD) or caspase-9 inhibitors (LEHD) did not attenuate TNF $\alpha$-induced necroptosis (Figure 6A). Similarly, addition of ROS inhibitors Tempol and NAC did not inhibit necroptosis in MVEC (Figure 6B). These data suggest that ROS or caspase-3 and -9 do not play significant roles in cardiac endothelial cell necroptosis. 
Figure 6

A.

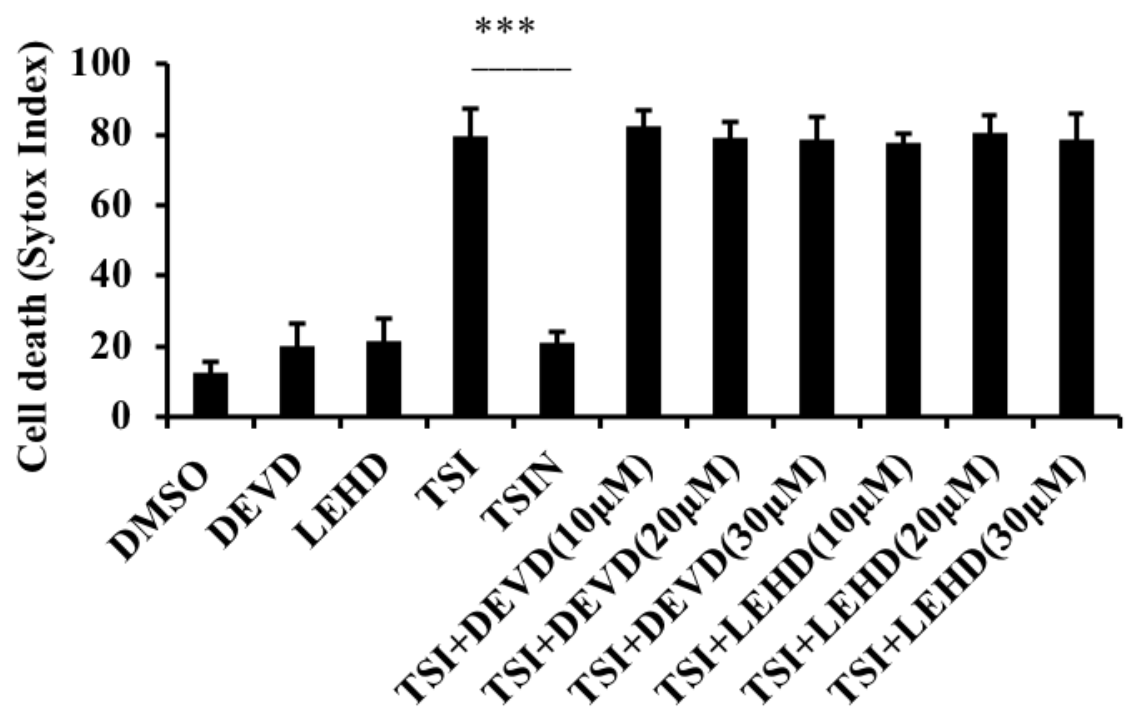

B.

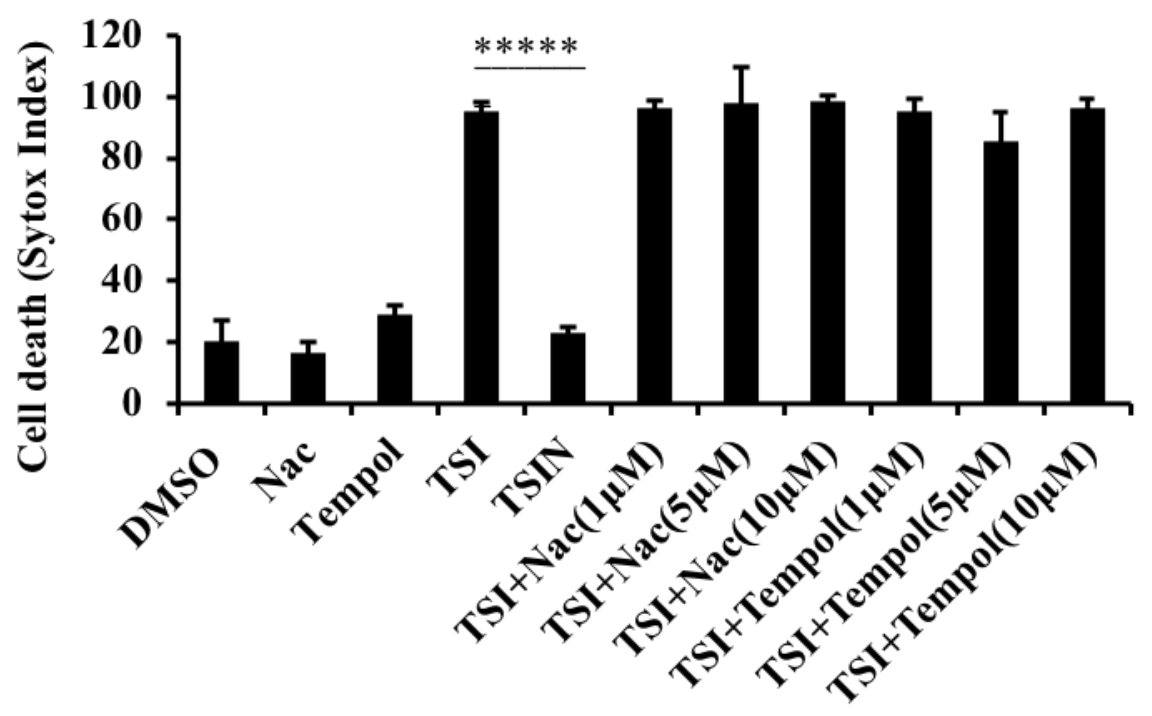


Figure 6. ROS, caspase-3 and caspase-9 do not participate in MVEC necroptosis.

(A) B6 MVEC were plated on 96 well plate in triplicates and treated with 100 $\mathrm{ng} / \mathrm{ml} \mathrm{hTNF} \alpha(\mathrm{T}), 100 \mathrm{nM}$ Smac mimetic (S) with or without $30 \mu \mathrm{M}$ IETD (I), $10 \mu \mathrm{M}$ Nec-1s $(\mathrm{N})$, caspase-3 inhibitor DEVD $(30 \mu \mathrm{M})$ or caspase-9 inhibitor LEHD $(30 \mu \mathrm{M})$. (B) ROS inhibitors NAC $(10 \mu \mathrm{M})$ and Tempol $(10 \mu \mathrm{M})$ were added to inhibit ROS activities. Cell death was quantified by SYTOX fluorescent intensity with IncuCyte ZOOM live imaging system. Data at 24 hours were shown as mean $\pm \mathrm{SD}$ and representative of 3 independent experiments. $* * * p<0.001, * * * * * p \leq 0.00001, \mathrm{t}$-test. 


\subsection{Cyp-D mediated necroptosis is linked to MLKL activation}

MLKL is phosphorylated by RIPK3 and then migrates to lipid rafts where it forms a pore, ultimately resulting in cell membrane rupture- a characteristic feature of necroptosis. ${ }^{6}$ To investigate if Cyp-D mediated necroptosis is linked to MLKL activation, MLKL inhibitor GW806742X was added and was observed to partially inhibit necroptosis in MVEC (Figure 7A). MLKL siRNA silencing in MVEC (Figures 7B\&C) prevented necroptotic cell death (Figure 7D).

Next, we analyzed expression of phosphorylated MLKL (pMLKL), which is the active form of MLKL, by western blot. pMLKL increased significantly in B6 MVEC under necroptosis inducing conditions, which can be inhibited by Cyp-D inhibitor CsA or by Cyp-D deficiency (Figure 7E), suggesting that Cyp-D is necessary for MLKL phosphorylation during necroptosis. 
Figure 7

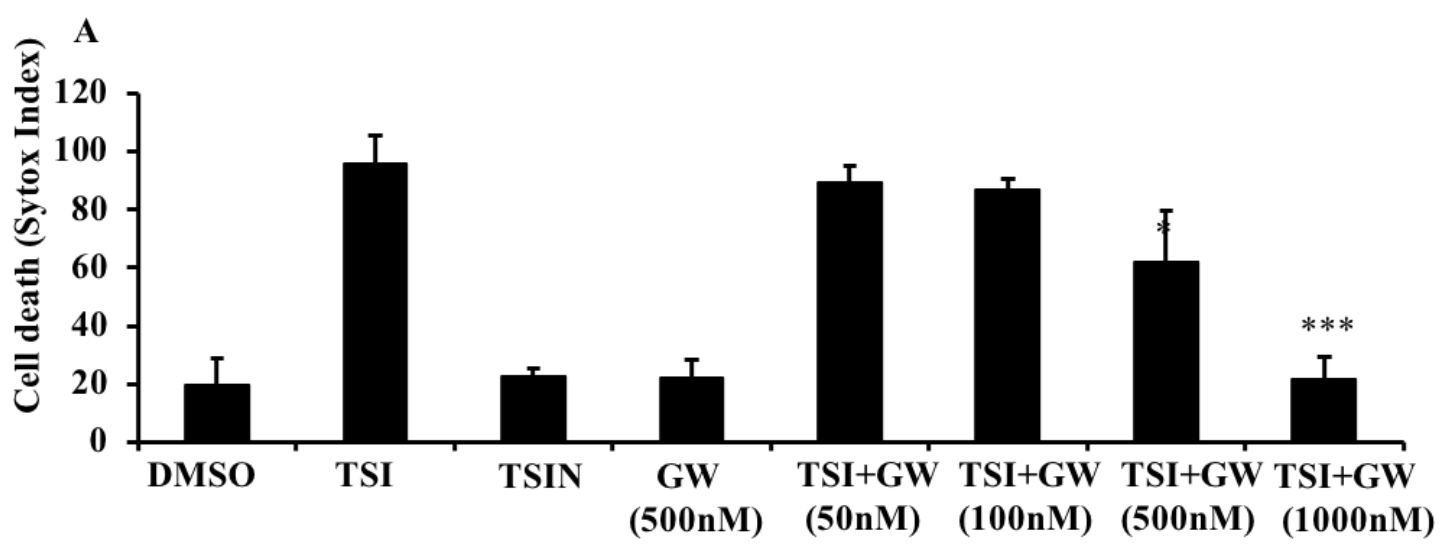

B. MLKL mRNA

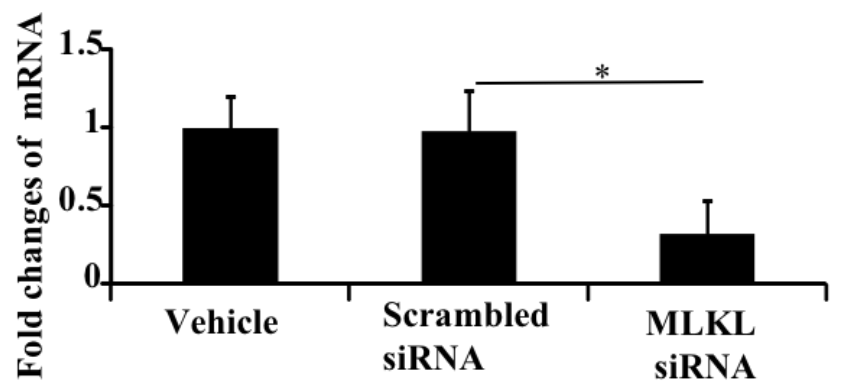

C. MLKL western blot

Scramble MLKL siRNA siRNA

Anti- $\beta$-actin
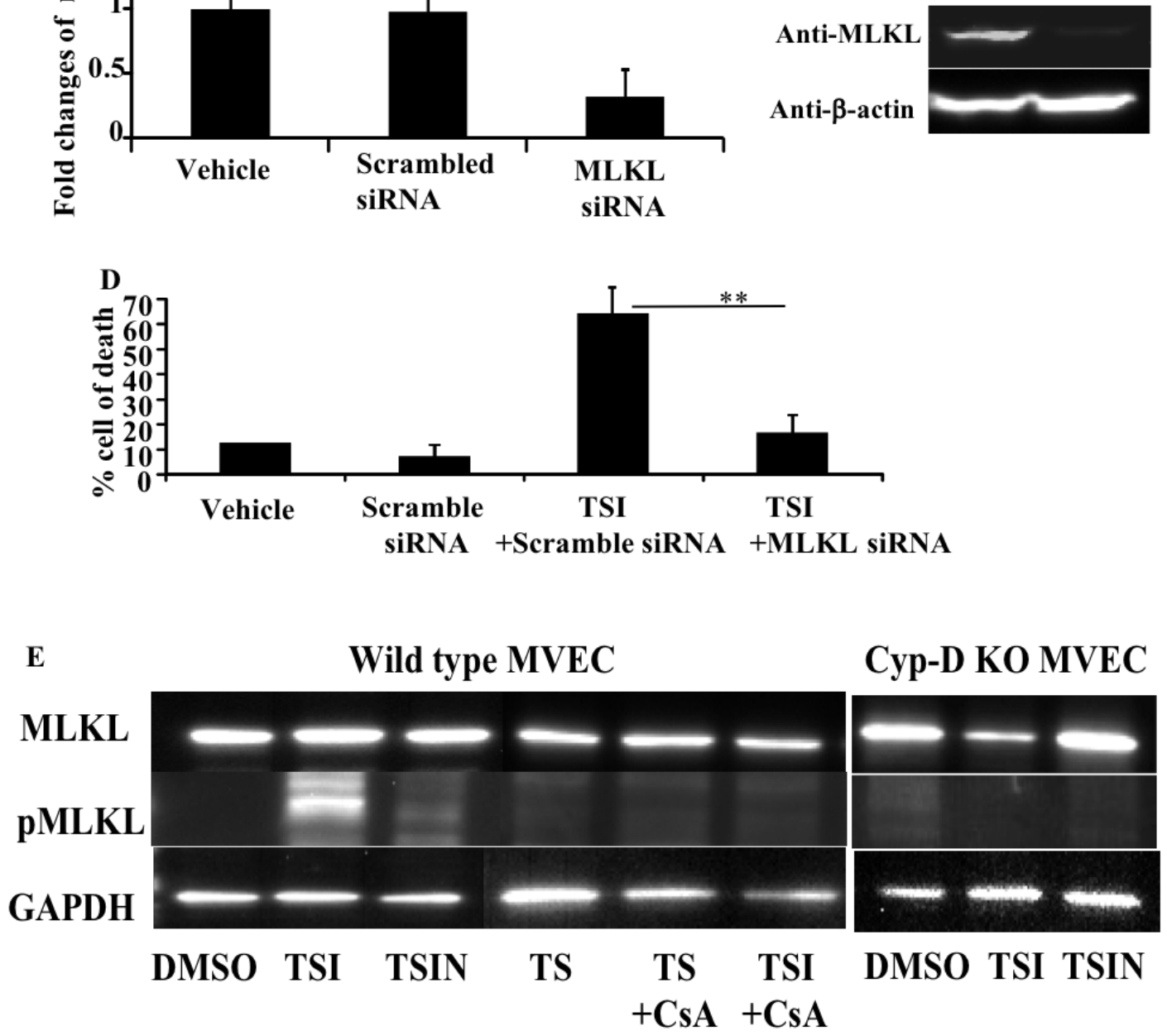
Figure 7. Cyp-D-regulated necroptosis is linked to downstream MLKL activation

(A) B6 MVEC necroptotic death was induced as in Figure 1. MLKL inhibitor GW806742X (GW, 50-1000 nM) was added. Cell death was quantified by SYTOX fluorescent intensity with IncuCyte ZOOM live imaging system. Data at 24 hours were shown as mean $\pm \mathrm{SD}$ and representative of 3 independent experiments. (B) MLKL was silenced by pooled MLKL siRNAs (Santa Cruz). MLKL knockdown was confirmed by real-time PCR 10 hours later. B-actin amplification was used as the control. Data at 24 hours were shown as mean $\pm \mathrm{SD}$ and representative of 3 independent PCR. (C) MLKL protein inhibition was confirmed 40 hours after by western blot using anti-total MLKL and loading control anti- $\beta$-actin. The same result was obtained in a repeated experiment. (D) MLKL knockdown protects MVEC from necroptotic death. 24 hours after siRNA silencing, necroptosis was induced in MVECs . Cell death was measured by 7-AAD positivity using flow cytometry. Data is a representative average of 3 independent cell death experiments. (E) Analysis of MLKL phosphorylation. Necroptosis was induced in B6 and Cyp-D ${ }^{-/}$MVEC as described in Figures 1-3. Cells were collected 6 hours later and cell lysates were analyzed for pMLKL by western blot using anti-phospho S345 MLKL. Antibodies against to total MLKL and house-keeping gene GAPDH were used as controls. ${ }^{*} \mathrm{p}<0.05$. $* * \mathrm{p}<0.01 . * * * \mathrm{p}<0.001$. 


\subsection{Cyp-D deficiency in donor cardiac grafts attenuates rejection}

Our data indicate that Cyp-D may be an effective target to inhibit cell death. To determine if Cyp-D deficiency in donor cardiac grafts can improve transplant survival, we performed heterotopic transplantation of wildtype B6 or Cyp- $\mathrm{D}^{-/-}$hearts into BALB/c mice followed by a brief period of immunosuppression spanning 0-9 days with sirolimus (rapamycin) (18). Cyp-D ${ }^{-/-}$grafts survived significantly longer post transplantation compared to wild type C57BL/6 grafts ( $89 \pm 43$ versus $29 \pm 6, n=7, p<0.0001)$ (Figure 8A).

To assess graft injury, we collected the cohort grafts 28 days post-transplantation. Naïve B6 hearts do not appear to be histologically different from naïve Cyp-D hearts (Figure 9A). B6 grafts showed severe lymphocyte infiltration and endothelial damage when compared to the Cyp- $\mathrm{D}^{-/}$grafts collected 28 days post-transplantation (Figures 9B\&C). However, tissue apoptosis was not significantly different between wild type and Cyp-D ${ }^{-/-}$grafts as defined by anti-cleaved caspase-3 immunohistochemistry staining (Figure 9D). In summary, these data suggest that Cyp-D deficiency protects cardiac grafts from rejection. 
Figure 8

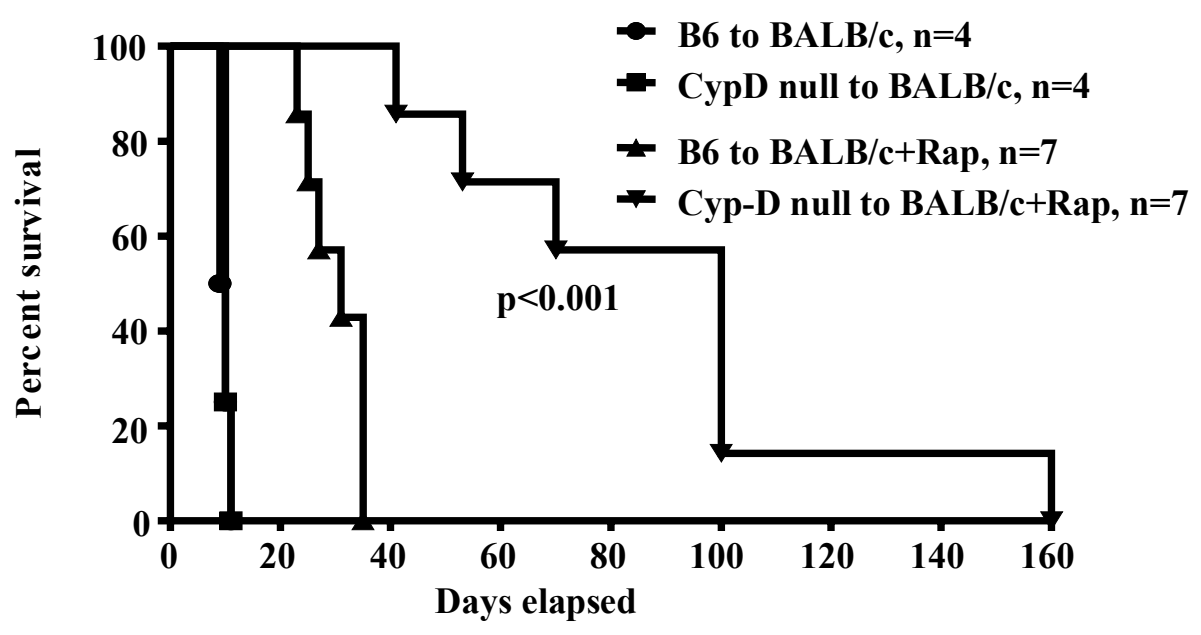

Figure 9

A.

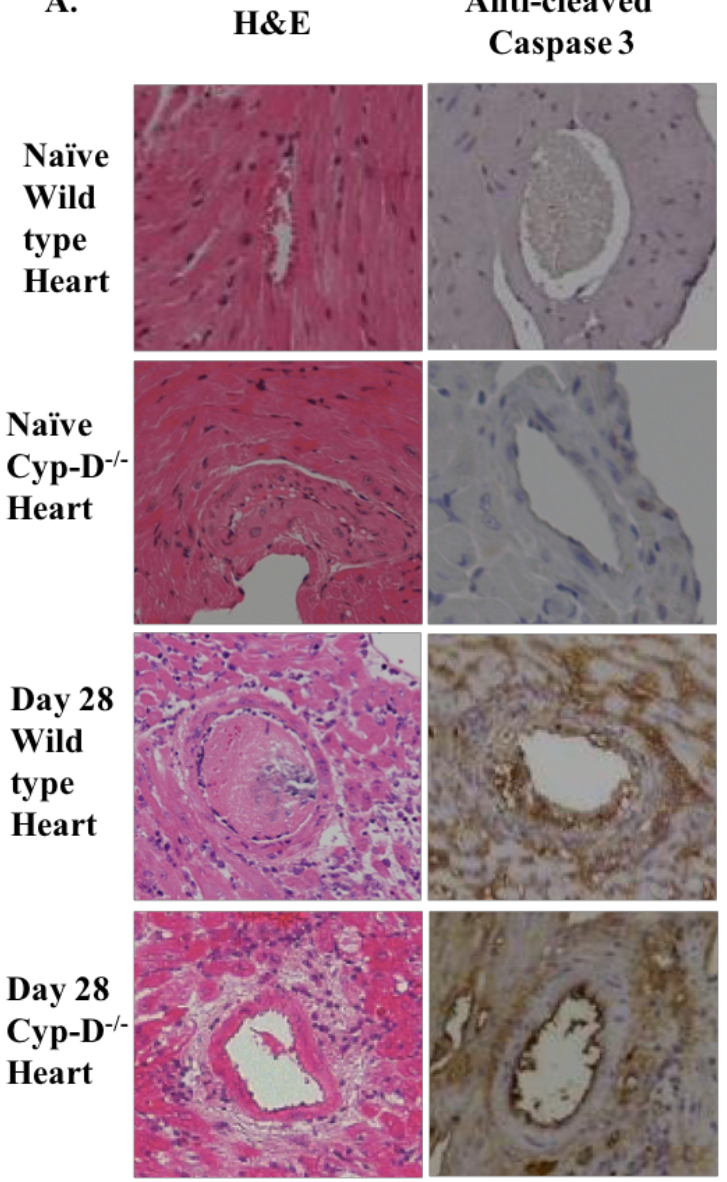

B.
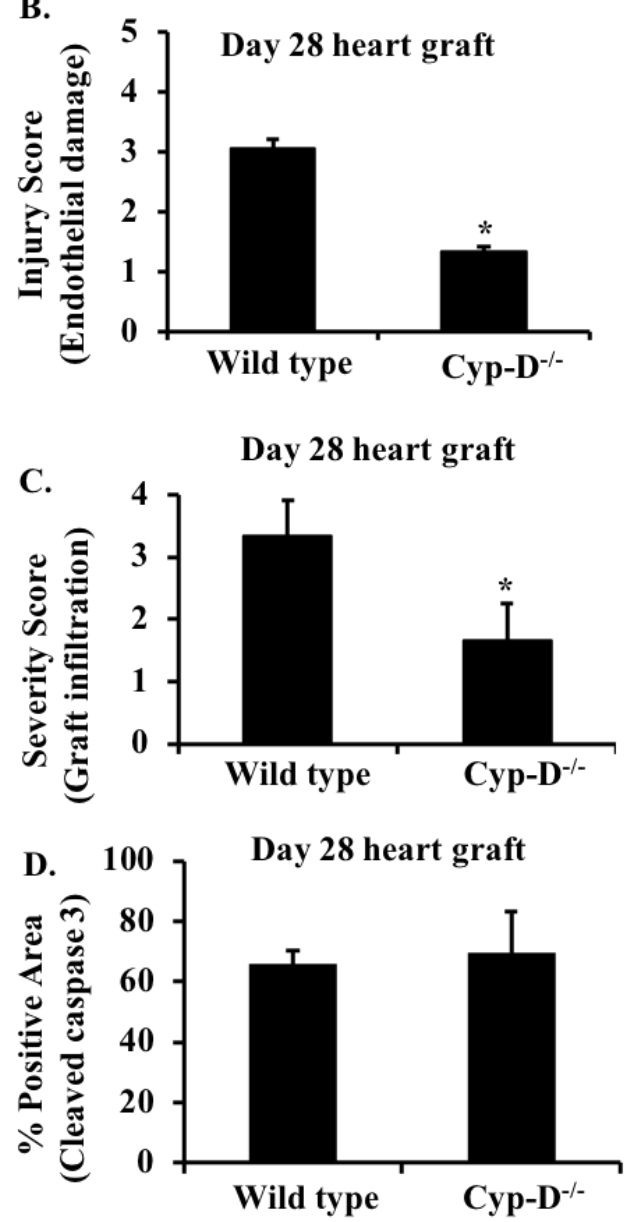
Figure 8. Cyp-D deficiency in donor cardiac grafts attenuated transplant rejection.

B6 or Cyp-D $\mathrm{D}^{-/}$cardiac grafts were heterotopically transplanted into abdomens of BALB/c mice, followed by rapamycin (Rap) treatment $(1 \mathrm{mg} / \mathrm{kg}$, day $0-9)$. Grafts were palpated and scored daily and considered rejected when pulsation ceased. $(n=4-7$, $* * * \mathrm{p}<0.001$, Mantel-Cox log-rank test).

Figure 9. Cyp-D deficiency in donor grafts inhibits endothelium damage.

(A) B6 or Cyp-D ${ }^{-/-}$cardiac grafts were transplanted into BALB/c mice as in Figure 5. Recipients ( $\mathrm{n}=4$ per group) were euthanized 28 days post-transplantation and grafts were collected for H\&E staining and anti-cleaved caspase-3 immunohistochemistry as detailed in the Methods. Microscope magnification=200 fold. Scale bar=100 $\mu \mathrm{M}$. (B) Endothelial damage and $(\mathbf{C})$ graft lymphocyte infiltration were scored blindly by a pathologist on a scale of $0=4$ ( 0 : no change, $1: 0-24 \%$ change, $2: 25-49 \%$ change; $3: 50$ $74 \%$ change, $4:>75 \%$ change). (D) Quantification of the cleaved caspase- 3 staining. Positive areas were automatically quantified by pooling all areas of slides (Nikon Eclipse). ${ }^{*} \mathrm{p}<0.05$, t-test. 
Figure 10

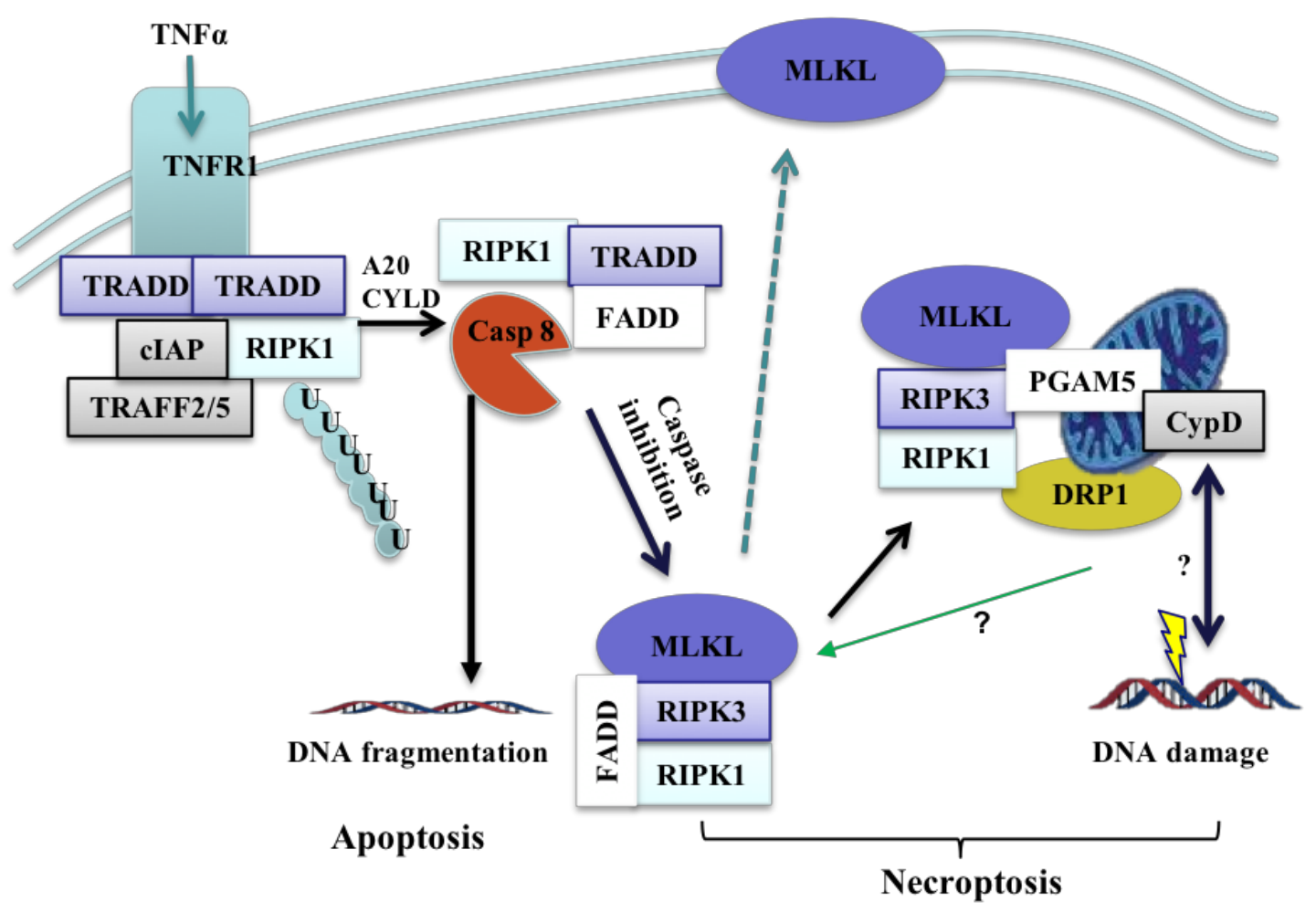

Figure 10. Proposed mechanism of cell death.

Upon stimulation of TNFR by TNF $\alpha$, TNFR associated death domain (TRADD) is recruited to the plasma membrane, which in turn recruits RIPK1, cellular inhibitors of apoptosis protein-1 and 2 (cIAP1/2), and TNF receptor-associated factor 2 and 5 (TRAF2/5) to form a receptor-bound complex I that initiates downstream signaling events. The deubiquitination of RIPK1 via A20 or Cylindromatosis (CYLD) activity causes complex I to dissociate from the membrane to form a cytosolic complex II with recruitment of FADD and caspase- 8 (Casp-8). Caspase- 8 will initiate apoptosis via cascades of caspases including caspase-3 (Casp-3), and cleave RIPK1 and RIPK3. Inhibition of caspase- 8 allows the formation of RIPK1-RIPK3 necrosome which recruits and phosphorylates MLKL that ultimately induces cell membrane rupture and necroptosis. Alternatively, the necrosome complex may also shuttle to the mitochondrial membrane to induce prolonged $\mathrm{mPTP}$ opening and mitochondrial dysfunction. This may promote downstream MLKL activation as well as release mitochondrial molecules that mediate DNA breakage. 


\section{Chapter 4}

\section{Discussion}

\subsection{Summary}

Solid organ transplantation remains a vital therapy for end stage heart failure. Historically, post-operative care was emphasized; however, the alleviation of cellular injury and subsequent inflammation gained more attention over the last few years. We have demonstrated previously that programmed cell death, particularly RIPK3-mediated necroptosis, can promote inflammation and tissue injury in donor heart and kidney grafts, and inhibition of RIPK3 attenuated graft rejection. ${ }^{64,70,71}$ However, necroptosis molecular pathways downstream of RIPK3 remain unknown. In this study, we demonstrated that mitochondria are the critical component in the downstream necroptotic pathway; specifically, inhibition of the mPTP regulator molecule Cyp-D attenuated MVEC necroptosis in vitro, and Cyp-D deficient cardiac allografts showed prolonged survival compared to wild-type C57BL/6 grafts post transplantation. Our study thus demonstrates that mPTP participates in RIPK3 mediated necroptosis and contributes to inflammatory injury in cardiac allografts, and that Cyp-D can be an important therapeutic target for long-term graft survival.

\subsection{MVECs necroptosis is mediated by mitochondrial dependent mechanisms}

It has been demonstrated the over the years that necroptosis is initiated by death receptors such as TNF $\alpha$, which leads to the downstream RIPK1/3 activation and formation of the necrosome. This complex has been proposed to induce necroptosis via mitochondrial independent or dependent pathways. ${ }^{30}$

The mitochondrial independent pathway involves MLKL mediated cellular membrane rupture. Upon its phosphorylation by RIPK3, MLKL undergoes conformational change, oligomerizes, and binds to plasma membrane lipids. Its $\mathrm{N}$ terminal domain then perforates the membrane and induces DAMPs release. More recently, MLKL has been additionally proposed to translocate to the mitochondria and to induce mitochondrial ROS production and mitochondrial permeability increase. ROS in 
turn promote necroptosis by activating RIPK1 autophosphorylation, therefore creating a positive feedback loop for necroptosis induction. ${ }^{72,73}$ In our study, however, ROS inhibition did not result in significant reduction in cell death (Fig 6B), suggesting that mitochondrial permeability may be a more significant contributor in death induction.

MLKL induced mitochondrial permeability increase induces cytochrome $c$ release, causing the formation of the supramolecular apoptosome complex with deoxyATP, APF1, and pro-caspase $9 .{ }^{72,73}$ Activated caspase 9 then activates the downstream executer, caspase 3. Caspase 3 is key in promoting cellular disassembly and nuclear fragmentation by activating CAD through inactivating the inhibitor of CAD (ICAD). ${ }^{74,75}$ Following translocation to the nucleus, CAD homodimerizes and creates double-stranded DNA breaks. In contrast, our study showed that inhibition of caspase 3 or 9 did not prevent necroptosis (Fig 6A). This suggests that other downstream mechanisms may be responsible for mitochondrial dependent necroptosis induction, such as mitochondrial molecules AIF and endonuclease G. ${ }^{76,77,78}$ As AIF and endonuclease G have been characterized as apoptogenic factors, it would be interesting to investigate whether they also participate in MVEC necroptosis.

\subsection{The role of Cyp-D in organ injury}

The involvement of mitochondria molecules as downstream RIPK mediators is still a point of contention. While it is a well-established fact that mitochondria are platforms for apoptosis execution, the prospect of direct mitochondrial involvement in programmed necrosis remains controversial, and contradictory experiment results point towards either mitochondrial-dependent or -independent forms of necroptosis. ${ }^{72}$ Our study demonstrates that Cyp-D, as an essential component of mitochondrial permeability transition regulator with an important role in apoptotic induction, also participates in necroptosis and thus may be an important therapeutic target.

Genetics experiments confirmed that targeting Cyp-D protected cells from necrosis, where CyP-D knockout in MEFs (mice embryonic fibroblast cells) resulted in a

partial rescue of necroptotic cell death ${ }^{57}$ in vitro, and that MPT pore is primarily involved in necrotic cell death instead of apoptosis. ${ }^{80} \mathrm{We}$ found that Cyp-D deficiency in donor cardiac grafts attenuated rejection and promoted long term survival. The fact that cleaved 
caspase 3 expression was not significantly different between WT and Cyp-D null grafts indicate that Cyp-D deficiency did not alter apoptotic tissue injury long-term. The data suggests that Cyp-D dependent mitochondrial permeability contributes to DAMPs release and augments inflammatory injury in the graft tissue.

\subsection{Mediators of RIPK1/3/MLKL and mitochondrial- dependent MVEC necroptosis}

Even though our data suggested that mitochondrial permeability transition and its regulator Cyp-D participates in necroptosis, the precise mechanism of MLKL participation in the MPTP pathway following TNF and caspase 8 inhibition is controversial. In Figure 7 we illustrated several possible MLKL pathways in the necroptotic pathway. After caspase 8 inhibition, it is known that MLKL translocates to the plasma membrane to induce membrane rupture and DAMP release. Alternatively, it has been proposed that RIPK3 recruits MLKL and mitochondrial protein phosphatase PGAM5 - a molecule suggested to be essential for mitochondrial fragmentation. PGAM5 recruits dynamin related protein Drp1 and dephosphorylates the serine 637 site of Drp1, activating its GTPase. ${ }^{60}$ Drp1 then translocate to mitochondrial outer membrane division sites and causes mitochondrial fragmentation. ${ }^{60}$

Drp1 activation has been shown to be involved in apoptosis, where under apoptotic stimuli, Drp1 induced Bax oligomerization and mitochondrial cytochrome $c$ release $^{81}$. Furthermore, Drp1 knockout Purkinje neurons displayed no signs of apoptotic death $^{82}$. Interestingly, Drp1 has also been implicated to play a role in necroptosis, where it regulates mitochondrial division. Recent studies demonstrated that Drp1 mediated mitochondrial division is mediated by RIPK 1 and RIPK $3^{60}$. Thus the RIPK3/MLKL/PGAM5/DRP1 is implicated to induce mitochondria - dependent necroptosis. However, the contributions of PGAM5 and DRP1 in the necroptotic pathway come under scrutiny as some studies suggest that PGAM5 and DRP1 may be dispensable. In our study, activated MLKL increased significantly in the necroptosis induced MVECs, but not in Cyp-D null MVECs, which suggests that Cyp-D activation is necessary for downstream MLKL phosphorylation events. We observed multiple banding for phosphorylated MLKL proteins, possibility due to the formation of MLKL multimers. Further studies are needed to determine if RIPK1/3/MLKL may directly activate Cyp-D, 
or if it is activated through indirect pathways - for instance, others have suggested that Cyp-D mediate Drp1 function and augment Drp1 recruitment to the mitochondria.

\subsection{Strategy to prevent graft injury in transplantation}

As novel immunosuppression methods have arisen over the past several years, strategies that target intra-graft death and inflammation still hold great potential for investigation. Currently, siRNA therapy has been utilized to target apoptotic molecules such as caspases 3 and 8 during IRI in mice. ${ }^{83}$ However, when used on porcine kidneys, caspase 3 siRNA delivery augmented inflammatory response and kidney tissue damage instead ${ }^{84}$ indicating a crucial need for a thorough knowledge of the complex interconnected pathway mechanisms of apoptosis and necroptosis.

The $\mathrm{mPT}$ pore and its regulators have been recognized as drug targets for IRI and neurodegenerative disorders, where Cyp-D knockout mice showed improved outcomes in disease models compared to wildtype mice. The Cyp-D inhibitor CsA is a potent immunosuppressive which disrupts calcineurin function and downstream immune cell activation. However, the 17 different subtypes of Cyp-D in the human genome remain poorly understood, and a comprehensive understanding of the similarities and differences between Cyp-D subgroups is necessary for specific and effective drug design. ${ }^{85}$ Nevertheless, it would be interesting to determine which Cyp-D family would the most effective target in the prevention of cell death without compromising mitochondrial function, and to investigate delivery of that specific Cyp-D shRNA via viral vectors into heart grafts to promote long-term tolerance.

\subsection{Limitations}

A limitation of the findings of this project is that the in vitro model may not accurately represent the subsequent in vivo transplantation results. First, the usage of small molecule inhibitors may yield multiple off-target effects. Second, the endothelial cell lines may not accurately reflect the histological results in two aspects: 1) complex pathways and immunological response may occur in vivo that are not seen in cell culture, such as immune-mediated attack of $\mathrm{T}$ cells ${ }^{86}$, and 2) there are limitations to our $\mathrm{KO}$ 
models, where regions of genetic variability may exist due to the presence of "flanking" genes incorporated into the genetic background ${ }^{87}$.

In terms of immunological study, it may be beneficial to do an initial investigation into how immune response regulates cell survival (i.e. apply mixed lymphocyte reaction), in addition to mitochondrial dysfunction in vitro beforehand, so as to better anticipate in vivo transplantation results. To improve upon our in vivo model, we may consider siRNA delivery into hearts ${ }^{88,89}$, although it is for short-term silencing and therefore may not be able to potentiate long-term protection in heart grafts post transplantation. CRISPR/cas9based strategy, on the other hand, may be a more promising area to explore. Alternatively, we may consider viral particle delivery technology to achieve high efficiency tissue delivery.

\subsection{Conclusion}

In this study, we demonstrated that mPTP plays an important role in necroptotic death of cardiac cells and graft rejection. Additionally, inhibition of Cyp-D attenuated RIPK3downstream MLKL phosphorylation, leading to prolonged survival when compared with wild type grafts. Our study shows that Cyp-D is an effective target in mitigating necroptosis in MVEC and preventing cardiac graft rejection. As Cyp-D deficiency prevents MPTP function without completely impeding mitochondrial function, targeting Cyp-D may offer exciting therapeutic potential in the prevention of long-term graft injury.

\subsection{Overall project significance}

Since long-term survival of transplants has not been greatly improved by posttransplant core strategies that solely target the immune response, a focus on understanding tissue injury as characterized by cell death induced inflammatory events may yield more effective strategies. Determining the role of mitochondria in regulated cell death pathways may be crucial to expanding our as-yet incomplete understanding of the downstream RIPK signaling in necroptosis. Therapeutically, we believe that targeting 
the mitochondria is a promising tactic in an effort to prevent regulated necrosis and ultimately, to promote transplant survival.

\subsection{Future Directions}

While it is known that mPT downstream mechanisms is classically associated with pro-apoptotic and necrotic stimuli, it is interesting that caspase 3 and ROS do not significantly contribute to MVEC death. This suggests that other mediators may be at play for necrotic DNA damage which is independent of caspase ROS, for instance, apoptosis-inducing factor and endonuclease G (EndoG). After released into the cytosol, AIF and EndoG translocate to the nucleus an is responsible for large scale DNA fragmentation. AIF and EndoG participate in apoptosis, but further investigation into their roles, or lack thereof, in necroptosis may also be warranted. Importantly, it will be crucial to translate our findings to clinically relevant study, by using a viral vector to deliver shRNA into heart grafts in order to inhibit Cyp-D and promote long-term survival. 


\section{References}

(1) Tonsho, M., Michel, S., Ahmed, Z et al. (2014). Heart Transplantation: Challenges Facing the Field. Cold Spring Harbor Perspectives in Medicine,4(5).

(2) Kittleson, M., Kobashigawa, J. (2017). Cardiac Transplantation: Current Outcomes and Comtemporary Controversies. Journal of the American College of Cardiology, 5(12).

(3) Pallet, N., Dieudé, M., Cailhier, J., \& Hébert, M. (2012). The Molecular Legacy of Apoptosis in Transplantation. American Journal of Transplantation,12(6), 13781384.

(4) Hébert, M., \& Jevnikar, A. M. (2015). The Impact of Regulated Cell Death Pathways on Alloimmune Responses and Graft Injury. Current Transplantation Reports,2(3), 242-258.

(5) Davidson, S. M., \& Duchen, M. R. (2007). Endothelial Mitochondria: Contributing to Vascular Function and Disease. Circulation Research, 100(8), 11281141.

(6) Peter, M. E. (2011). Programmed cell death: Apoptosis meets necrosis. Nature, 471(7338), 310-312.

(7) Taylor, R. C., Cullen, S. P., \& Martin, S. J. (2008). Apoptosis: Controlled demolition at the cellular level. Nature Reviews Molecular Cell Biology,9(3), 231241.

(8) Berghe, T. V., Linkermann, A., Jouan-Lanhouet, S., Walczak, H., \& Vandenabeele, P. (2014). Regulated necrosis: The expanding network of nonapoptotic cell death pathways. Nature Reviews Molecular Cell Biology,15(2), 135147.

(9) Fulda, S., \& Debatin, K. (2006). Extrinsic versus intrinsic apoptosis pathways in anticancer chemotherapy. Oncogene,25(34), 4798-4811.

(10) Tsujimoto, Y., \& Shimizu, S. (2006). Role of the mitochondrial membrane permeability transition in cell death. Apoptosis, 12(5), 835-840.

(11) Zhan, M., Brooks, C., Liu, F., Sun, L., \& Dong, Z. (2013). Mitochondrial dynamics: Regulatory mechanisms and emerging role in renal pathophysiology. Kidney International,83(4), 568-581.

(12) Schwarz, M., Andrade-Navarro, M. A., \& Gross, A. (2007). Mitochondrial carriers and pores: Key regulators of the mitochondrial apoptotic program? Apoptosis, 12(5), 869-876.

(13) Daisy, P., \& Saipriya, K. (2012). BCL-2 Family Proteins: The Mitochondrial Apoptotic Key Regulators. Current Cancer Therapy Reviews, 8(2), 133-140.

(14) Yang, S., Zhao, X., Xu, H., Chen, F., Xu, Y., Li, Z., . . Ye, J. (2017). AKT2 Blocks Nucleus Translocation of Apoptosis-Inducing Factor (AIF) and Endonuclease G (EndoG) While Promoting Caspase Activation during Cardiac Ischemia. International Journal of Molecular Sciences, 18(3), 565.

(15) Fiers, W., Beyaert, R., Declercq, W., \& Vandenabeele, P. (1999). More than one way to die: Apoptosis, necrosis and reactive oxygen damage. Oncogene, 18(54), 77197730 .

(16) Brenner, D., Blaser, H., \& Mak, T. W. (2015). Regulation of tumour necrosis factor signalling: Live or let die. Nature Reviews Immunology, 15(6), 362-374.

(17) Rastogi, S., Rizwani, W., Joshi, B., Kunigal, S., \& Chellappan, S. P. (2011). TNF$\alpha$ response of vascular endothelial and vascular smooth muscle cells involve 
differential utilization of ASK1 kinase and p73. Cell Death \& Differentiation,19(2), 274-283.

(18) Dhuriya, Y. K., \& Sharma, D. (2018). Necroptosis: A regulated inflammatory mode of cell death. Journal of Neuroinflammation,15(1).

(19) Yuan, J. (2015). Faculty of 1000 evaluation for Caspase- 8 regulates TNF- $\alpha$ induced epithelial necroptosis and terminal ileitis. F1000 - Post-publication Peer Review of the Biomedical Literature.

(20) Xie, Y., Hou, W., Song, X., Yu, Y., Huang, J., Sun, X., . . Tang, D. (2016). Ferroptosis: Process and function. Cell Death \& Differentiation,23(3), 369-379.

(21) Fearnhead, H. O., Vandenabeele, P., \& Berghe, T. V. (2017). How do we fit ferroptosis in the family of regulated cell death? Cell Death and Differentiation, 24(12), 1991-1998.

(22) Sato, M., Kusumi, R., Hamashima, S., Kobayashi, S., Sasaki, S., Komiyama, Y., . . S Sato, H. (2018). The ferroptosis inducer erastin irreversibly inhibits system $\mathrm{xc}-$ and synergizes with cisplatin to increase cisplatin's cytotoxicity in cancer cells. Scientific Reports, 8(1).

(23) Lewerenz, J., Ates, G., Methner, A., Conrad, M., \& Maher, P. (2018). Oxytosis/Ferroptosis - (Re-) Emerging Roles for Oxidative Stress-Dependent Nonapoptotic Cell Death in Diseases of the Central Nervous System. Frontiers in Neuroscience, 12.

(24) David, K. K. (2009). Parthanatos, a messenger of death. Frontiers in Bioscience, Volume(14), 1116. doi:10.2741/3297

(25) Shin, H., Kwon, H., Lee, J., Gui, X., Achek, A., Kim, J., \& Choi, S. (2015). Doxorubicin-induced necrosis is mediated by poly-(ADP-ribose) polymerase 1 (PARP1) but is independent of p53. Scientific Reports, 5(1).

(26) Fatokun, A. A., Dawson, V. L., \& Dawson, T. M. (2014). Parthanatos: Mitochondrial-linked mechanisms and therapeutic opportunities. British Journal of Pharmacology, 171(8), 2000-2016.

(27) Andrabi, S. A., Dawson, T. M., \& Dawson, V. L. (2008). Mitochondrial and Nuclear Cross Talk in Cell Death. Annals of the New York Academy of Sciences, 1147(1), 233-241.

(28) Caja, S., \& Enríquez, J. A. (2017). Mitochondria in endothelial cells: Sensors and integrators of environmental cues. Redox Biology,12, 821-827.

(29) Schwarz, M., Andrade-Navarro, M. A., \& Gross, A. (2007). Mitochondrial carriers and pores: Key regulators of the mitochondrial apoptotic program? Apoptosis, 12(5), 869-876.

(30) Thornton, C., \& Hagberg, H. (2015). Role of mitochondria in apoptotic and necroptotic cell death in the developing brain. Clinica Chimica Acta; International Journal of Clinical Chemistry, 451(Pt A), 35-38.

(31) Tsujimoto, Y. (1998). Role of Bcl-2 family proteins in apoptosis: Apoptosomes or mitochondria? Genes to Cells,3(11), 697-707.

(32) Kutuk, O., \& Basaga, H. (2006). Bcl-2 protein family: Implications in vascular apoptosis and atherosclerosis. Apoptosis, 11(10), 1661-1675.

(33) Nicotra, A., \& Parvez, S. (2002). Apoptotic molecules and MPTP-induced cell death. Neurotoxicology and Teratology,24(5), 599-605.

(34) Kwong, J., \& Molkentin, J. (2015). Physiological and Pathological Roles of the Mitochondrial Permeability Transition Pore in the Heart. Cell Metabolism,21(2), 206214. 
(35) Karch, J., \& Molkentin, J. D. (2014). Identifying the components of the elusive mitochondrial permeability transition pore. Proceedings of the National Academy of Sciences, 111(29), 10396-10397.

(36) Lemasters, J. J., Theruvath, T. P., Zhong, Z., \& Nieminen, A.-L. (2009). Mitochondrial Calcium and the Permeability Transition in Cell Death. Biochimica et Biophysica Acta, 1787(11), 1395-1401. http://doi.org/10.1016/j.bbabio.2009.06.009

(37) Pastorino, J. G., Tafani, M., Rothman, R. J., Marcineviciute, A., Hoek, J. B., \& Farber, J. L.(1999). Functional Consequences of the Sustained or Transient Activation by Bax of the Mitochondrial Permeability Transition Pore. Journal of Biological Chemistry, 274(44), 31734-31739

(38) Daisy, P., \& Saipriya, K. BCL-2 Family Proteins: The Mitochondrial Apoptotic

Key Regulators. Current Cancer Therapy Reviews, 2012; 8(2), 133-140.

(39) Pastorino, J. G., Tafani, M., Rothman, R. J., Marcineviciute, A., Hoek, J. B., \& Farber, J. L. Functional Consequences of the Sustained or Transient Activation by Bax of the Mitochondrial Permeability Transition Pore. Journal of Biological Chemistry, 1999; 274(44), 31734-31739.

(40) Karch, J., Kwong, J. Q., Burr, A. R., Sargent, M. A., Elrod, J. W., Peixoto, P. M., ... Molkentin, J. D. (2013). Bax and Bak function as the outer membrane component of the mitochondrial permeability pore in regulating necrotic cell death in mice. ELife, 2. doi:10.7554/elife.00772

(41) Tischner, D., Manzl, C., Soratroi, C., Villunger, A., \& Krumschnabel, G. Necrosis-like death can engage multiple pro-apoptotic Bcl-2 protein family members. Apoptosis, 2012; 17(11), 1197-1209.

(42) Lomonosova, E., \& Chinnadurai, G. BH3-only proteins in apoptosis and beyond: an overview. 2008; Oncogene,27.

(43) Gauba, E., Guo, L., \& Du, H. (2017). Cyclophilin D Promotes Brain Mitochondrial F1FO ATP Synthase Dysfunction in Aging Mice. Journal of Alzheimer's Disease: JAD, 55(4), 1351-1362.

(44) Thomas, B., Banerjee, R., Starkova, N. N., Zhang, S. F., Calingasan, N. Y., Yang, L., ... Starkov, A. (2012). Mitochondrial Permeability Transition Pore Component Cyclophilin D Distinguishes Nigrostriatal Dopaminergic Death Paradigms in the MPTP Mouse Model of Parkinson's Disease. Antioxidants \& Redox Signaling, 16(9), 855-868.

(45) Schneider, M. D. (2005). Cyclophilin D: Knocking On Deaths Door. Science Signaling,2005(287).

(46) Linkermann A, Brasen J, Darding, H, et al. Two independent pathways of regulated necrosis mediate ischemia-reperfusion injury. Proc. Natl. Acad. Sci. U.S.A. 2013; 110: 12024-12029

(47) Beutner, G., Alanzalon, R. E., \& Porter, G. A. (2017). Cyclophilin D regulates the dynamic assembly of mitochondrial ATP synthase into synthasomes. Scientific Reports, 7, 14488.

(48) Beck, S. J., Guo, L., Phensy, A., Tian, J., Wang, L., Tandon, N., . . . Du, H. (2016). Deregulation of mitochondrial F1FO-ATP synthase via OSCP in Alzheimer's disease. Nature Communications, 7, 11483.

(49) Kim, S. Y., Shim, M. S., Kim, K.-Y., Weinreb, R. N., Wheeler, L. A., \& Ju, W.K. (2014). Inhibition of cyclophilin D by cyclosporin A promotes retinal ganglion cell survival by preventing mitochondrial alteration in ischemic injury. Cell Death \& Disease, 5(3), e1105-. 
(50) Kwong, J., \& Molkentin, J. (2015). Physiological and Pathological Roles of the Mitochondrial Permeability Transition Pore in the Heart. Cell Metabolism,21(2), 206214.

(51) Nacev, B. A., Low, W.-K., Huang, Z., Su, T. T., Su, Z., Alkuraya, H., ... Liu, J. O. (2011). A Calcineurin-Independent Mechanism of Angiogenesis Inhibition by a Nonimmunosuppressive Cyclosporin A Analog. The Journal of Pharmacology and Experimental Therapeutics, 338(2), 466-475.

(52) Liu J, Farmer JDJ, Lane WS, Friedman J, Weissman I, Schreiber SL. Calcineurin is a common target of cyclophilin-cyclosporin A and FKBP-FK506 complexes. Cell. 1991;66:807-815.

(53) Vanlangenakker, N, Vanden Berghe, T, Bogaert P, et al. cIAP and TAK1 protect cells from TNF-induced necrosis by preventing RIP1/RIP3 dependent reactive oxygen species production. Cell Death Differ. 18, 656-665

(54) Zhan M, Brooks, C, Liu, F, et al. Mitochondrial dynamics: regulatory mechanisms and emerging role in renal pathophysiology. Kidney Int. 2013; 83(4): $568-581$

(55) Holley, A. K., Dhar, S. K., Xu, Y., \& St. Clair, D. K. (2012). Manganese superoxide dismutase: beyond life and death. Amino Acids, 42(1), 139-158.

(56) Brouns, S.J., Jore, M.M, Lundgren M. et al. Small CRISPR RNA guide antiviral defense in prokaryotes. Science 321, 960-964

(57) Karch J, Kanisicak O, Brody M, et al. Necroptosis interfaces with MOMP and the MPTP in mediating cell death PLoS ONE 2015; 10(6) e 0130520

(58) Tsujimto Y, and Shimizu S. Role of mitochondrial membrane permeability transition in cell death. Apoptosis 2007; 12:835-840

(59) Bains, CP, Kaiser, RA, Purcell, NH, et al. Loss of cyclophilin D reveals a critical role for mitochondrial permeability transition in cell death. Nature. 2005 ; 434: 658662

(60) Wang Z, Jiang H, Chen S, et al. The mitochondrial phosphatase PGAM5 functions at the convergence point of multiple necrotic death pathways. Cell. 2012; 148: $228-243$

(61) Zhang L, Jiang F, Chen Y, et al. Necrostatin-1 attenuates ischemia injury induced cell deth in rat tubular cell line NRK-52E through decreased DRP1 expression. Int. J. Mol. Sci. 2013; 14: 24742-24754

(62) Tait SW, Oberst A, Quarato G, et al. Widespread mitochondrial depletion via mitophagy does not compromise necroptosis. Cell Rep. 2013; 5(4): 878-85

(63) Lim S. Y., Davidson S. M., Mocanu M. M., Yellon D. M., Smith C. C. (2007). The cardioprotective effect of necrostatin requires the cyclophilin-D component of the mitochondrial permeability transition pore. Cardiovasc. Drugs Ther. 21, 467-469 10.1007/s10557-007-6067-6

(64) Pavlosky A, Lau A, Su Y, Lian D, Huang X, Yin Z et al. RIPK3-Mediated Necroptosis Regulates Cardiac Allograft Rejection. Am J Transplant 2014;14(8):1778-1790.

(65) Kawashima S, Yamamoto T, Horiuchi Y, Fujiwara K, Gouda S, Yoshimura Y et al. S-15176 and its methylated derivative suppress the CsA-insensitive mitochondrial permeability transition and subsequent cytochrome c release induced by silver ion, and show weak protonophoric activity. Mol Cell Biochem 2011;358(1-2):45-51 
(66) Elimadi A, Jullien V, Tillement JP, Morin D. S-15176 inhibits mitochondrial permeability transition via a mechanism independent of its antioxidant properties. Eur J Pharmacol 2003;468(2):93-101.

(67) Baines CP, Kaiser RA, Purcell NH, Blair NS, Osinska H, Hambleton MA et al. Loss of cyclophilin D reveals a critical role for mitochondrial permeability transition in cell death. Nature 2005;434(7033):658-662.

(68) Vanden Berghe T, Vanlangenakker N, Parthoens E, Deckers W, Devos M, Festjens $\mathrm{N}$ et al. Necroptosis, necrosis and secondary necrosis converge on similar cellular disintegration features. Cell Death Differ 2010;17(6):922-930.

(69) Shindo R, Kakehashi H, Okumura K, Kumagai Y, Nakano H. Critical contribution of oxidative stress to TNFalpha-induced necroptosis downstream of RIPK1 activation. Biochem Biophys Res Commun 2013;436(2):212-216.

(70) Lau A, Wang S, Jiang J, Haig A, Pavlosky A, Linkermann A et al. RIPK3mediated necroptosis promotes donor kidney inflammatory injury and reduces allograft survival. Am J Transplant 2013;13(11):2805-2818.

(71) Kwok C PA, Lian D, Jiang J, Huang X, Yin Z, Liu W, Haig A, Jevnikar A, Zhang , ZX. Necroptosis is Involved in CD4+ T-cell Mediated Microvascular Endothelial Cell Death and Chronic Cardiac Allograft Rejection. Transplantation 2017;101(9):2026-2037.

(72) Marshall KD, Baines CP. Necroptosis: is there a role for mitochondria? Front Physiol 2014;5:323.

(73) Kinnally KW, Peixoto PM, Ryu SY, Dejean LM. Is mPTP the gatekeeper for necrosis, apoptosis, or both? Biochim Biophys Acta 2011;1813(4):616-622.

(74) Li LY, Luo X, Wang X. Endonuclease G is an apoptotic DNase when released from mitochondria. Nature 2001;412(6842):95-99.

(75) Bahi N, Zhang J, Llovera M, Ballester M, Comella JX, Sanchis D. Switch from caspase-dependent to caspase-independent death during heart development: essential role of endonuclease $\mathrm{G}$ in ischemia-induced DNA processing of differentiated cardiomyocytes. J Biol Chem 2006;281(32):22943-22952.

(76) Baritaud M, Boujrad H, Lorenzo HK, Krantic S, Susin SA. Histone H2AX: The missing link in AIF-mediated caspase-independent programmed necrosis. Cell Cycle 2010;9(16):3166-3173.

(77) Cabon L, Galan-Malo P, Bouharrour A, Delavallee L, Brunelle-Navas MN, Lorenzo HK et al. BID regulates AIF-mediated caspase-independent necroptosis by promoting BAX

(78) Li Z, Fan EK, Liu J, Scott MJ, Li Y, Li S et al. Cold-inducible RNA-binding protein through TLR4 signaling induces mitochondrial DNA fragmentation and regulates macrophage cell death after trauma. Cell Death Dis 2017;8(5):e2775.

(79) Wang H, Sun L, Su L, Rizo J, Liu L, Wang LF et al. Mixed lineage kinase domain-like protein MLKL causes necrotic membrane disruption upon phosphorylation by RIP3. Mol Cell 2014;54(1):133-146.

(80) Bains, CP, Kaiser, RA, Purcell, NH, et al. Loss of cyclophilin D reveals a critical role for mitochondrial permeability transition in cell death. Nature. 2005; 434: 658662

(81) Borner, C. (n.d.). Faculty of 1000 evaluation for Membrane remodeling induced by the dynamin-related protein Drp1 stimulates Bax oligomerization. F1000 - Postpublication peer review of the biomedical literature. 
(82) Kageyama, Y., Zhang, Z., Roda, R., Fukaya, M., Wakabayashi, J., Wakabayashi, N., . . Sesaki, H. Mitochondrial division ensures the survival of postmitotic neurons by suppressing oxidative damage. 2012; The Journal of Cell Biology, 197(4), 535-551.

(83) Zhang, X. et al. (2006). Prevention Of Renal Ischemic Injury By Silencing The Expression Of Renal Caspase 3 And Caspase 8. Transplantation,82(Suppl 2), 307.

(84) Yang B, Hosgood SA, Nicholson ML. Naked small interfering RNA of caspase-3 in preservation solution and autologous blood perfusate protects isolated ischemic porcine kidneys. Transplan- tation 2011; 91: 501-507.

(85) Davis, T. L., Walker, J. R., Campagna-Slater, V., Finerty, P. J., Paramanathan, R., Bernstein, G., ... Dhe-Paganon, S. (2010). Structural and Biochemical Characterization of the Human Cyclophilin Family of Peptidyl-Prolyl Isomerases. PLoS Biology, 8(7), e1000439.

(86) Pietra BA, Wiseman A, Bolwerk A, et al. CD4 T cell-mediated cardiac allograft rejection requires donor but not host MHC class II. J Clin Invest. 2000; 106(8): 100310

(87) Eisener-Dorman AF, Lawrence DA, Bolivar VJ. Cautionary insights on knockout mouse studies: the gene or not the gene?. Brain Behav Immun. 2008;23(3):318-24.

(88) Zhang, Z., Beduhn, M. E., Zheng, X., Min, W., \& Jevnikar, A. M. Preventing Tissue Injury Using siRNA. RNA Interference Methods in Molecular Biology, 2010; 341-355.

(89) Zheng, X., Lian, D., Wong, A., Bygrave, M., Ichim, T. E., Khoshniat, M., . . Min, W. Novel Small Interfering RNA-Containing Solution Protecting Donor Organs in Heart Transplantation. Circulation, 2009; 120(12), 1099-1107. 


\section{Curriculum Vitae}

Name:

Post-secondary Education and Degrees:

Honours and Awards:

\section{Related Work Experience:}

Publications:
Ingrid Gan

2009-2014 The University of Western Ontario London, Ontario, Canada Hon. Spec. Biology

2009-2012 The University of Western Ontario London, Ontario, Canada Piano Performance Diploma

$2017 \quad 2^{\text {nd }}$ place oral presentation winner Infection and Immunity Research Forum, UWO

2016 Poster of distinction recipient American Transplant Congress, Chicago

2014 Dean's Honor List, UWO

2010 Fred Pattison Piano Competition winner, UWO
Research Assistant

The University of Western Ontario 2016

Gan I, Jiang J, Lian D, et al. Mitochondrial permeability regulates cardiac endothelial cell necroptosis and cardiac allograft rejection. Am J Transplant. 2018;00:1-13.

Zhang, Z.-X., Gan, I., Pavlosky, A., Huang, X., Fuhrmann, B., \& Jevnikar, A. M. (2017). Intracellular pH Regulates TRAIL-Induced Apoptosis and Necroptosis in Endothelial Cells. Journal of Immunology Research, 2017, 1503960. 
Platform presentations:
Mitochondrial membrane permeability plays a critical role for endothelial cell necroptosis and cardiac allograft rejection.

Canadian Society of Transplantation 2016 Annual Scientific Meeting, Sep 27 ${ }^{\text {th }}$, 2017, Halifax, Nova Scotia

Mitochondrial permeability regulates endothelial cell necroptotic death and cardiac allograft rejection. Infection and Immunity Research Forum, Oct $27^{\text {th }}, 2016$, Western University

Mitochondrial permeability regulates endothelial cell necroptotic death and cardiac allograft rejection. Canadian Society of

Transplantation 2016 Annual Scientific Meeting, Oct 16 ${ }^{\text {th }}, 2016$, Quebec City, Quebec 